Comment. Math. Helv. 73 (1998) 353-378

(C) 1998 Birkhäuser Verlag, Basel

$0010-2571 / 98 / 030353-26 \$ 1.50+0.20 / 0$

Commentarii Mathematici Helvetici

\title{
The representation ring of a compact Lie group revisited
}

\author{
Bob Oliver
}

\begin{abstract}
We describe a new construction of the induction homomorphism for representation rings of compact Lie groups: a homomorphism first defined by Graeme Segal. The idea is to first define the induction homomorphism for class functions, and then show that this map sends characters to characters. This requires a detection theorem - a class function of $G$ is a character if its restrictions to certain subgroups of $G$ are characters - which in turn requires a review of the representation theory for nonconnected compact Lie groups.
\end{abstract}

Mathematics Subject Classification (1991). Primary: 22E45; secondary: 55R40, 55R50.

Keywords. Induced representations, class functions.

In his 1968 paper, Segal [Seg] used elliptic operators to construct induction homomorphisms $\mathrm{R}(H) \rightarrow \mathrm{R}(G)$ for an arbitrary pair $H \subseteq G$ of compact Lie groups, and then applied this to prove (among other things) a detection result for when a class function on $G$ is a character. In this paper, we give new proofs of these results, but in the reverse order. We begin in Section 1 by showing that a class function on $G$ is a character if its restrictions to all finite subgroups of $G$ are characters. Then, in Section 2, we first define induction homomorphisms $\mathrm{Cl}(H) \rightarrow$ $\mathrm{Cl}(G)$ for class functions, and afterwards apply the results of Section 1 to show that they send characters to characters and hence define induction maps between the representation rings. This gives a construction of the induction homomorphisms which is more elementary than that of Segal (though also less elegant), in that it only assumes the standard theory of representations of a compact connected Lie group.

It is the results in Section 3 which, while more technical, provided the original motivation for this work. Let $\mathcal{S}_{\mathcal{P}}(G)$ be the family of all $p$-toral subgroups of $G$ (for all primes $p$ ), where a group is called $p$-toral if it is an extension of a torus by a finite $p$-group. Let $\mathrm{R}_{\mathcal{P}}(G)$ be the inverse limit of the representation rings $\mathrm{R}(P)$ for all $P \in \mathcal{S}_{\mathcal{P}}(G)$, where the limit is taken with respect to restriction and conjugation in $G$. This group $\mathrm{R}_{\mathcal{P}}(G)$ was shown in [JO, Theorem 1.8] to be isomorphic to the Grothendieck group $\mathbb{K}(B G)$ of the monoid of vector bundles over $B G$; and the 
"restriction" homomorphism

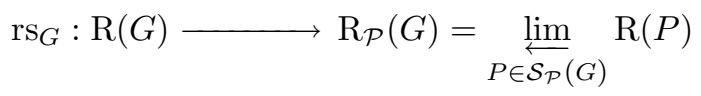

is isomorphic to the natural homomorphism $\mathrm{R}(G) \rightarrow \mathbb{K}(B G)$ which sends a representation $V$ to the bundle $\left(E G \times{ }_{G} V\right) \downarrow B G$.

The main result of Section 3 is a description, for arbitrary $G$, of the cokernel of the homomorphism $\mathrm{rs}_{G}$. In particular, we show that it is onto whenever $G$ is finite or $\pi_{0}(G)$ is a $p$-group; but that it is not surjective in general. Precise necessary and sufficient conditions for $\mathrm{rs}_{G}$ to be onto are given in Theorem 3.10, and several simpler sufficient conditions are given in Corollary 3.11. Note that $\mathrm{rs}_{G}$ is surjective if and only if bundles over $B G$ have the following property: for each $\xi \downarrow B G$ there exist $G$-representations $V, V^{\prime}$ such that $\xi \oplus\left(E G \times{ }_{G} V^{\prime}\right) \cong\left(E G \times{ }_{G} V\right)$ (since by [JO, Theorem 1.8], every bundle over $B G$ is a summand of a bundle coming from a $G$-representation).

In the above discussion, we have for simplicity dealt only with the complex representation rings. But most of the results are shown below for real as well as complex representations.

I would like to thank in particular Stefan Jackowski for his comments and suggestions about this work. Originally, Sections 1 and 3 were intended to go into our joint paper [JO], but then they grew to the point where we decided to publish them separately. I would also like to thank the colleague who, at the 1996 summer research institute in Seattle, showed me the references [Ta] and [Vo] on representation theory for nonconnected compact Lie groups. (After that conference, I asked several people if they were the ones who had done so, but they all denied it.)

\section{Section 1. Detection of characters}

The main results of this section are Propositions 1.2 and 1.5: on detecting characters among class functions. They follow from Proposition 1.4, which describes the representation theory of nonconnected compact Lie groups. The first part of Proposition 1.4 - the bijection between irreducible $G$-representations and certain irreducible representations of $N_{G}(T, C)$ - was proven by Takeuchi [Ta, Theorem 4], and is also stated in [Vo, Theorem 1.17]. Since their notation is very different from that used here, we have found it simplest to keep our proof, rather than just refer to [Ta]. Note that the group which we call $N=N_{G}(T, C)$ is denoted $T$ in $[\mathrm{Ta}]$ and $[\mathrm{Vo}]$ (and called the Cartan subgroup in [Vo]).

Throughout this section, $G$ denotes a fixed compact Lie group, and $G_{0}$ is its identity connected component. Fix a maximal torus $T \subseteq G$, let $W_{G}=N_{G}(T) / T$ denote its Weyl group, and let $\mathfrak{t} \subseteq \mathfrak{g}$ denote the Lie algebras of $T$ and $G$. For any Weyl chamber $C \subseteq \mathfrak{t}$, define

$$
N_{G}(T, C)=\left\{g \in N_{G}(T) \mid \operatorname{Ad}(g)(C)=C\right\},
$$


and

$$
N_{G}(T, \pm C)=\left\{g \in N_{G}(T) \mid \operatorname{Ad}(g)( \pm C)=( \pm C)\right\}
$$

Here, $\operatorname{Ad}(g)$ denotes the adjoint (conjugation) action of $g$ on $\mathfrak{t}$ and $\mathfrak{g}$. We will see in Proposition 1.1 that $N_{G}(T, C)$ has exactly one connected component for each connected component of $G$, and that every element of $G$ is conjugate to an element of $N_{G}(T, C)$. Then, in Proposition 1.2 below, we show that a (continuous) class function $f \in \mathrm{Cl}(G)$ is a character of $G$ if and only if $f \mid N_{G}(T, C)$ is a character, and that $f$ is a real character of $G$ (i.e., the character of a virtual $\mathbb{R} G$-representation) if and only if $f \mid N_{G}(T, \pm C)$ is a real character. At the same time, we construct (Proposition 1.4) a one-to-one correspondence between the irreducible representations of $G$, and those irreducible representations of $N_{G}(T, C)$ whose weights lie in the dual Weyl chamber $C^{*}$. This generalizes the standard relationship, for a connected compact Lie group $G$, between the irreducible representations of $G$ and those of $T$.

Afterwards, the detection result is extended to show that an element $f \in \mathrm{Cl}(G)$ is a character (real character) if and only if $f \mid H$ is a character (real character) of $H$ for each finite subgroup $H \subseteq G$. The classical theorem of Brauer for detecting characters on finite groups can then be applied to further restrict the class of finite subgroups of $G$ which have to be considered.

We first recall the definition and basic properties of the Weyl chambers of a compact connected Lie group $G$. The set of irreducible representations (or irreducible characters) of $T$ will be identified here with $T^{*} \stackrel{\text { def }}{=} \operatorname{Hom}\left(T, S^{1}\right)$; which will in turn be regarded as a lattice in $\mathfrak{t}^{*}=\operatorname{Hom}(\mathfrak{t}, \mathbb{R})$.

The roots of $G$ (or of $G_{0}$ ) are the characters of the nontrivial irreducible summands of the adjoint representation of $T$ on $\mathbb{C} \otimes_{\mathbb{R}} \mathfrak{g}$. They occur in pairs $\pm \theta$. Let $R \subseteq T^{*} \subseteq \mathfrak{t}^{*}$ denote the set of roots of $G$. Any element $x_{0} \in \mathfrak{t}$ such that $\theta\left(x_{0}\right) \neq 0$ for all $\theta \in R$ determines a choice of positive roots

$$
R_{+}=\left\{\theta \in R \mid \theta\left(x_{0}\right)>0\right\} .
$$

And this in turn determines a Weyl chamber

$$
C=\left\{x \in \mathfrak{t} \mid \theta(x) \geq 0 \forall \theta \in R_{+}\right\} \subseteq \mathfrak{t}
$$

and a dual Weyl chamber

$$
C^{*}=\left\{x \in \mathfrak{t}^{*} \mid\langle\theta, x\rangle \geq 0 \forall \theta \in R_{+}\right\} \subseteq \mathfrak{t}^{*} .
$$

Here, in the definition of $C^{*},\langle-,-\rangle$ denotes any $G$-invariant inner product on $\mathfrak{g}^{*}$. Note that $C^{*}$ is independent of the choice of inner product, since a $G$-invariant inner product is uniquely defined up to scalar on each simple component of $G$.

Proposition 1.1. Fix a maximal torus $T \subseteq G$ and a Weyl chamber $C \subseteq \mathfrak{t}$, and set $N=N_{G}(T, C)$. Then $N \cap G_{0}=T, N \cdot G_{0}=G$, and hence $N / T \cong G / G_{0}$. Also, any element of $G$ is conjugate to an element of $N$. 
Proof. Recall that the Weyl group $W_{G_{0}}=N_{G_{0}}(T) / T$ of $G_{0}$ permutes the Weyl chambers of $T$ simply and transitively (cf. [Ad, Lemma 5.13]). Hence each coset of $N_{G_{0}}(T)$ in $N_{G}(T)$ contains exactly one connected component of $N=N_{G}(T, C)$; and so $N \cap G_{0}=T, N \cdot G_{0}=G$, and $N / T \cong G / G_{0}$.

By [Bo, $\S 5.3$, Theorem 1(b)], any automorphism of $G_{0}$ leaves invariant some maximal torus and some Weyl chamber in $G_{0}$. Hence, any element $g \in G$ is contained in $N_{G}\left(T^{\prime}, C^{\prime}\right)$ for some maximal torus $T^{\prime}$ and some Weyl chamber $C^{\prime} \subseteq T^{\prime}$; and $T^{\prime}$ and $T$ are conjugate in $G_{0}$ (cf. [Ad, Corollary 4.23]). Since $N_{G_{0}}(T) / T$ permutes the Weyl chambers for $T$ transitively, there is $a \in G_{0}$ such that $T=a T^{\prime} a^{-1}$ and $C=a C^{\prime} a^{-1}$; and $a g a^{-1} \in N=N_{G}(T, C)$.

When dealing with real representations, we need to distinguish between the different types of irreducible representations and characters. As usual, we say that a $G$-representation $V$ (over $\mathbb{C}$ ) has real type if it has the form $V \cong \mathbb{C} \otimes_{\mathbb{R}} V^{\prime}$ for some $\mathbb{R} G$-representation $V^{\prime}$; and that $V$ has quaternion type if it is the restriction of an $\mathbb{H} G$-representation. If $V$ is irreducible and its character is real-valued, then $V$ has real or quaternion type, but not both [Ad, Proposition 3.56]. By a real character will be meant the character of a virtual representation of real type (i.e., the difference of two representations of real type).

Proposition 1.2. Fix a maximal torus $T \subseteq G$ and a Weyl chamber $C \subseteq \mathfrak{t}$. Then a continuous class function $f: G \rightarrow \mathbb{C}$ is a character of $G$ if and only if $f \mid N_{G}(T, C)$ is a character of $N_{G}(T, C)$. And a continuous class function $f: G \rightarrow \mathbb{R}$ is a real character of $G$ if and only if $f \mid N_{G}(T, \pm C)$ is a real character.

The proof of Proposition 1.2 will be given after that of Proposition 1.4 below. We first note some elementary conditions for $f$ to be a character or a real character. In the following lemma, we write as usual $\langle\varphi, \psi\rangle=\int_{G} \varphi(g) \overline{\psi(g)}$ for any pair of continuous functions $\varphi, \psi: G \rightarrow \mathbb{C}$ (where the integral is the Haar integral on $G$ with measure 1).

Lemma 1.3. (a) A class function $f \in \mathrm{Cl}(G)$ is a character of $G$ if and only if $\langle f, \chi\rangle \in \mathbb{Z}$ for each character $\chi$ of $G$.

(b) A class function $f: G \rightarrow \mathbb{R}$ is a real character of $G$ if and only if $f$ is a character, and $\left\langle f, \chi_{V}\right\rangle \in 2 \mathbb{Z}$ for each $G$-representation $V$ of quaternion type.

(c) A class function $f: G \rightarrow \mathbb{R}$ is a real character of $G$ if $f$ is a character, and $f \mid H$ is a real character of $H$ for some $H \triangleleft G$ of finite odd index.

Proof. For any pair $W, V$ of complex $G$-representations,

$$
\left\langle\chi_{W}, \chi_{V}\right\rangle=\operatorname{dim}_{\mathbb{C}}\left(\left(W^{*} \otimes_{\mathbb{C}} V\right)^{G}\right)=\operatorname{dim}_{\mathbb{C}}\left(\operatorname{Hom}_{\mathbb{C} G}(W, V)\right) \in \mathbb{Z} .
$$

(Recall that $\chi_{W^{*}}(g)=\overline{\chi_{W}(g)}$ for all $g \in G$.) Also, if $W$ has real type and $V$ 
has quaternion type, then $\operatorname{Hom}_{\mathbb{C} G}(W, V)$ is a quaternion vector space, and so its complex dimension is even. This proves the "only if" parts of points (a) and (b).

Conversely, assume that $f \in \mathrm{Cl}(G)$ is such that $\langle f, \chi\rangle \in \mathbb{Z}$ for each character $\chi$ of $G$. Since the irreducible characters form an orthonormal set, we know that $\langle f, f\rangle \geq \sum_{i=1}^{k}\left\langle f, \chi_{i}\right\rangle^{2}$ for any set $\chi_{1}, \ldots, \chi_{k}$ of distinct irreducible characters. Since each $\langle f, \chi\rangle \in \mathbb{Z}$, this shows that $\langle f, \chi\rangle=0$ for all but finitely many irreducible chararcters $\chi$; and so $f=\sum_{\chi}\langle f, \chi\rangle \cdot \chi$ is a character of $G$ by the Peter-Weyl theorem (cf. [Ad, Theorem 3.47]).

We now consider conditions for a real valued character to be a real character; or equivalently for a self-adjoint representation to be of real type. An irreducible $G$-representation (over $\mathbb{C}$ ) is of complex type if its character is not real valued; i.e., if $V \neq V^{*}$. It follows from [Ad, Theorem 3.57] that a $G$-representation $V$ (over $\mathbb{C}$ ) is of real type if and only if it is a sum of irreducible representations of real type, of representations $\mathbb{C} \otimes_{\mathbb{R}} W \cong W \oplus W^{*}$ for $W$ irreducible of complex type, and of representations $\mathbb{C} \otimes_{\mathbb{R}} W \cong W \oplus W$ for $W$ irreducible of quaternion type. If $v=\sum_{i=1}^{k} n_{i}\left[V_{i}\right] \in \mathrm{R}(G)$ has real valued character, where the $V_{i}$ are distinct irreducible $G$-representations, then $\sum_{i=1}^{k} n_{i}\left[V_{i}\right]=\sum_{i=1}^{k} n_{i}\left[\left(V_{i}\right)^{*}\right]$, and so each pair $V_{i},\left(V_{i}\right)^{*}$ occurs with the same multiplicity. Hence $v$ has real type if $2 \mid n_{i}$ for each $i$ such that $V_{i}$ has quaternion type. Since $n_{i}=\left\langle\chi_{v}, \chi_{V_{i}}\right\rangle$, this proves point (b).

It remains to prove point (c): that an element $v \in \mathrm{R}(G)$ with real valued character has real type if $v \mid H$ has real type for some normal subgroup $H \triangleleft G$ of finite odd index; we may assume that $v$ is the class of an actual $\mathbb{C}[G]$-representation $V$. Since all irreducible $\mathbb{C}[G / H]$-representations, aside from the trivial one, have complex type (cf. [Ser, Exercise 13.12]), we can write $\mathbb{C}[G / H] \cong \mathbb{C} \oplus W \oplus W^{*}$ for some representation $W$. Since by assumption, $V \mid H$ has real type and $V^{*} \cong V$, the isomorphism

$$
\operatorname{Ind}_{H}^{G}(V \mid H) \cong \mathbb{C}[G / H] \otimes_{\mathbb{C}} V \cong V \oplus\left(W \otimes_{\mathbb{C}} V\right) \oplus\left(W \otimes_{\mathbb{C}} V\right)^{*} \cong V \oplus \mathbb{C} \otimes_{\mathbb{R}}\left(W \otimes_{\mathbb{C}} V\right)
$$

shows that $V$ has real type.

By a weight of the compact Lie group $G$ is meant an element of the lattice $T^{*} \subseteq \mathfrak{t}^{*}$, regarded as an irreducible character of $T$. If $V$ is any representation of $G$, then the set of "weights of $V$ " is defined to be the set of characters of irreducible components of $V \mid T$. Consider the partial ordering of the weights of $G$, where $\phi_{1} \leq \phi_{2}$ if $\phi_{1}$ is contained in the convex hull of the $W_{G}$-orbit of $\phi_{2}$ (cf. [Ad, Definition 6.23]). One of the basic theorems of representation theory says that if $G$ is connected, then any irreducible $G$-representation $V$ has a unique $W_{G \text {-orbit of }}$ highest (maximal) weights, each of which occurs with multiplicity one. Furthermore, distinct irreducible representations have distinct orbits of higher weights, and every weight of $G$ can be realized as the highest weight of some irreducible 
$G$-representation. Thus, the irreducible representations of any connected $G$ are in one-to-one correspondence with the $W_{G}$-orbits of weights of $G$. And since any given dual Weyl chamber $C^{*} \subseteq \mathfrak{t}^{*}$ contains exactly one element in each $W_{G}$ orbit in $\mathrm{t}^{*}$ (cf. [Ad, Corollary 5.16]), the irreducible representations of $G$ are in one-to-one correspondence with the weights in $C^{*}$. For more detail, see, e.g., [Ad, Theorem 6.33] or [BtD, Section VI.2].

Now assume that $G$ is not connected. If $V$ is an irreducible $G$-representation, and if $V_{0}$ is any irreducible component of $V \mid G_{0}$, then $V$ is an irreducible summand of $\operatorname{Ind}_{G_{0}}^{G}\left(V_{0}\right)$. Hence each irreducible summand of $V \mid G_{0}$ is obtained from $V_{0}$ by conjugation by some element of $\pi_{0}(G)$; and there is still a uniquely defined $W_{G^{-}}$ orbit of highest weights for $V$. In this case, however, the highest weights can occur with multiplicity greater than one; and there can be several irreducible $G$ representations with the same orbit of highest weights.

In the next proposition, $\operatorname{Irr}(G)$ will denote the set of irreducible representations of $G$. Also, if $N=N_{G}(T, C)$ (for any maximal torus $T \subseteq G$ and any Weyl chamber $C \subseteq \mathfrak{t})$, then $\operatorname{Irr}\left(N, C^{*}\right)$ denotes the set of irreducible representations of $N$ whose weights all lie in the dual Weyl chamber $C^{*}$ of $C$. For any $V \in \operatorname{Irr}(G)$, $\operatorname{mx}_{C^{*}}(V) \subseteq C^{*} \cap T^{*}$ denotes the set of those maximal weights of the irreducible summands of $V \mid G_{0}$ which lie in $C^{*}$. And for any $N$-invariant set of weights $\Phi \subseteq T^{*}, V\langle\Phi\rangle$ denotes the sum of all irreducible summands of $V \mid T$ with weights in $\Phi$, regarded as an $N$-representation.

Proposition 1.4. Fix a maximal torus $T \subseteq G$ and a Weyl chamber $C \subseteq \mathfrak{t}$, and set $N=N_{G}(T, C)$. For any irreducible $G$-representation $V$, the subspace $V\left\langle\operatorname{mx}_{C^{*}}(V)\right\rangle$ is always an irreducible summand of $V \mid N$ having multiplicity one. This induces a bijection

$$
\beta_{G}: \operatorname{Irr}(G) \stackrel{\cong}{\longrightarrow} \operatorname{Irr}\left(N, C^{*}\right) \quad \text { defined by } \quad \beta_{G}([V])=\left[V\left\langle\operatorname{mx}_{C^{*}}(V)\right\rangle\right]
$$

and an isomorphism

$$
\bar{\beta}_{G}: \mathrm{R}(G) \stackrel{\cong}{\longrightarrow}\left(N, C^{*}\right) \quad \text { defined by } \quad \bar{\beta}_{G}([V])=\left[V\left\langle C^{*}\right\rangle\right] .
$$

Proof. Fix an irreducible $G_{0}$-representation $V_{0}$, and let $\phi$ be the maximal weight of $V_{0}$ lying in $C^{*}$. Set $\Phi=(N / T) \cdot \phi \subseteq C^{*}$, the $N / T$-orbit of $\phi$. Let $\left(V_{0}\right) \subseteq$ $\operatorname{Irr}\left(G_{0}\right)$ denote the $G / G_{0}$-orbit of $V_{0}$, and let $\operatorname{Irr}\left(G,\left(V_{0}\right)\right)$ denote the set of all irreducible $G$-representations with support in $\left(V_{0}\right)$; i.e., the set of those irreducible $G$-representations $V$ such that all irreducible summands of $V \mid G_{0}$ lie in $\left(V_{0}\right)$.

Let $V_{\phi}$ denote the (1-dimensional) irreducible representation with weight (character) $\phi$; regarded as a subspace of $V_{0}$. Since $G / G_{0} \cong N / T$, the uniqueness of maximal weights in $C^{*}$ shows that each irreducible component of $\left(\operatorname{Ind}_{G_{0}}^{G}\left(V_{0}\right)\right) \mid G_{0}$ contains exactly one weight in $\Phi=(N / T) \cdot \phi$ (and with multiplicity one). Thus,

$$
V_{0}\langle\Phi\rangle=V_{\phi} \quad \text { and } \quad \operatorname{Ind}_{G_{0}}^{G}\left(V_{0}\right)\langle\Phi\rangle=\operatorname{Ind}_{T}^{N}\left(V_{\phi}\right) .
$$


So for any $G$-representation $V^{\prime}$ with support in $\left(V_{0}\right)$ (i.e., for any $\left[V^{\prime}\right] \in \operatorname{Irr}\left(G,\left(V_{0}\right)\right)$ ), there is a commutative diagram

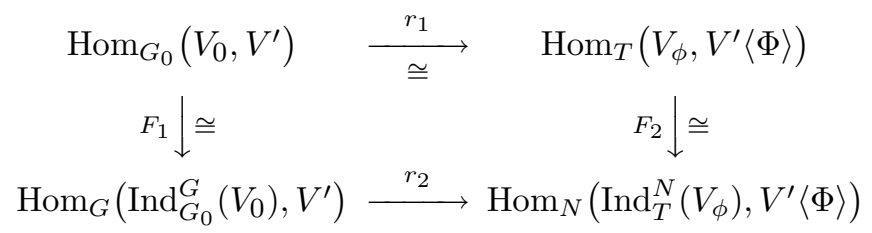

where $F_{1}$ and $F_{2}$ are the Frobenius reciprocity isomorphisms, and $r_{1}$ and $r_{2}$ are defined by restriction to summands with weights in $\Phi$. The one-to-one correspondence between irreducible $G_{0}$-representations and highest weights contained in $C^{*}$ shows that $r_{1}$ is an isomorphism, and thus that $r_{2}$ is also an isomorphism.

Now assume that $V$ and $V^{\prime}$ are two irreducible $G$-representations with support in $\left(V_{0}\right)$. By Frobenius reciprocity again $\left(\operatorname{Hom}_{G}\left(\operatorname{Ind}_{G_{0}}^{G}\left(V_{0}\right), V\right) \cong \operatorname{Hom}_{G_{0}}\left(V_{0}, V\right) \neq\right.$ $0), V$ is a summand of $\operatorname{Ind}_{G_{0}}^{G}\left(V_{0}\right)$. So by $(3)$ and $(4)$, for any $[V],\left[V^{\prime}\right] \in \operatorname{Irr}\left(G,\left(V_{0}\right)\right)$,

$$
\operatorname{Hom}_{N}\left(V\langle\Phi\rangle, V^{\prime}\langle\Phi\rangle\right) \cong \operatorname{Hom}_{G}\left(V, V^{\prime}\right) \cong \begin{cases}\mathbb{C} & \text { if } V \cong W \\ 0 & \text { if } V \neq W .\end{cases}
$$

This shows that $V\langle\Phi\rangle$ is $N$-irreducible for any $[V] \in \operatorname{Irr}\left(G,\left(V_{0}\right)\right)$, and that $V\langle\Phi\rangle \cong$ $V^{\prime}\langle\Phi\rangle$ if and only if $V \cong V^{\prime}$. And finally, any irreducible $N$-representation with support in $\Phi$ is a summand of $\operatorname{Ind}_{T}^{N}\left(V_{\phi}\right) \cong \operatorname{Ind}_{G_{0}}^{G}\left(V_{0}\right)\langle\Phi\rangle$, and hence has the form $V\langle\Phi\rangle$ for some $V \in \operatorname{Irr}\left(G_{0},\left(V_{0}\right)\right)$.

We have now shown that $\beta_{\Phi}: \operatorname{Irr}\left(G,\left(V_{0}\right)\right) \stackrel{\cong}{\longrightarrow} \operatorname{Irr}(N, \Phi)$, defined by setting $\beta_{\Phi}([V])=[V\langle\Phi\rangle]$, is a well defined bijection. Since the restriction to $G_{0}$ of any irreducible $G$-representation is a sum of representations in just one $G / G_{0}$-orbit of irreducible $G_{0}$-representations, $\beta_{G}: \operatorname{Irr}(G) \rightarrow \operatorname{Irr}\left(N, C^{*}\right)$ is the disjoint union of the $\beta_{\Phi}$ taken over all $N / T$-orbits $\Phi \subseteq\left(C^{*} \cap T^{*}\right)$ and hence also a bijection. This proves point (1). At the same time, it shows that the homomorphism $\bar{\beta}_{G}$ : $\mathrm{R}(G) \rightarrow \mathrm{R}\left(N, C^{*}\right)$ of $(2)$ is an isomorphism, since its matrix with respect to the bases of irreducible representations is triangular with 1's along the diagonal.

We are now ready to prove that a class function is a (real) character if its restriction to $N_{G}(T, C)\left(N_{G}(T, \pm C)\right)$ is a (real) character.

Proof of 1.2. Complex case: Fix a continuous class function $f: G \rightarrow \mathbb{C}$ such that $f \mid N$ is a character of $N$. We must show that $f$ is a character of $G$. Let $v_{0} \in \mathrm{R}(N)$ be such that $\chi_{v_{0}}=f \mid N$, let $\chi$ be the character of $\bar{\beta}_{G}^{-1}\left(v_{0}\left\langle C^{*}\right\rangle\right) \in \mathrm{R}(G)$ (Proposition 1.4(2)), and set $f^{\prime}=f-\chi$. By construction, $f^{\prime} \mid N$ is the character of an element $v \in \mathrm{R}(N)$ such that $v\left\langle C^{*}\right\rangle=0$. We will show that $v=0$. It then follows that $f^{\prime}=0$ (since every element of $G$ is conjugate to an element of $N$ ), and hence that $f=\chi$ is a character of $G$. 
Fix any $\phi \in T^{*}$, and let $N_{\phi} \subseteq N$ denote the subgroup of elements fixing $\phi$. Choose any $\psi \in \operatorname{interior}\left(C^{*}\right)^{N}\left(N / T\right.$ acts linearly on $\mathfrak{t}^{*}$ and leaves the dual Weyl chamber $C^{*}$ invariant). Then $\phi+\mathbb{R} \psi$ is not contained in the wall of any dual Weyl chamber (since $\psi$ is not); and so there is a dual Weyl chamber $C_{1}^{*}$ such that $\phi+\epsilon \psi \in \operatorname{interior}\left(C_{1}^{*}\right)$ for small $\epsilon>0$. Let $w \in W_{G}$ be any element such that $w\left(C_{1}^{*}\right)=C^{*}\left(W_{G_{0}}\right.$ permutes the Weyl chambers transitively). Then $w \phi \in C^{*}$, since $\phi \in C_{1}^{*}$. Also, for any $a \in N_{\phi}, a(\psi)=\psi$ and $a(\phi)=\phi$ by assumption, so $a$ leaves $C_{1}^{*}$ invariant, and hence $w a w^{-1}$ leaves $C^{*}=w\left(C_{1}^{*}\right)$ invariant. Thus $w N_{\phi} w^{-1} \subseteq N$; and so $v\langle w \phi\rangle=0 \in \mathrm{R}\left(w N_{\phi} w^{-1}\right)$ since $v\left\langle C^{*}\right\rangle=0 \in \mathrm{R}(N)$. Since $\chi_{v}$ is constant on $G$-conjugacy classes (it is the restriction of a class function on $G)$, it now follows that $v\langle\phi\rangle=0 \in \mathrm{R}\left(N_{\phi}\right)$.

Let $\phi_{1}, \ldots, \phi_{k} \in T^{*}$ be $N / T$-orbit representatives for the support of $v$, and write $N_{i}=N_{\phi_{i}}$ (the subgroup of elements which fix $\left.\phi_{i}\right)$. Then $v=\sum_{i=1}^{k} \operatorname{Ind}_{N_{i}}^{N}\left(v\left\langle\phi_{i}\right\rangle\right)$. We have just seen that $v\left\langle\phi_{i}\right\rangle=0 \in \mathrm{R}\left(N_{i}\right)$ for each $i$, and hence $v=0$.

Real case: Write $N_{ \pm}=N_{G}(T, \pm C)$, for short. Fix a class function $f: G \rightarrow \mathbb{C}$ such that $f \mid N_{ \pm}$is a real character. Then $f$ is a character by the above, and $f(G) \subseteq \mathbb{R}$ since any element of $G$ is conjugate to an element of $N \subseteq N_{ \pm}$(Proposition 1.1). By Lemma 1.3(b), we can assume (after replacing $f$ by its sum with an appropriate real character) that $f=\chi_{V}$, where $V=\sum_{i=1}^{k} V_{i}$, the $V_{i}$ are distinct irreducible $G$-representations of quaternion type, and $V \mid N_{ \pm}$is a representation of real type. We claim that $V=0$ (i.e., that $k=0$ ).

Assume otherwise: that $k>0$. Choose a $W_{G}$-orbit $\Psi$ of maximal weights in one of the $V_{i}$ - say $V_{1}$ - which does not occur in any of the others except possibly as maximal weights. Set $\Phi=\Psi \cap C^{*}$ and $\Phi_{ \pm}=\Psi \cap\left( \pm C^{*}\right)$. By Proposition 1.4 (and the original assumption on $\Psi), V_{1}\langle\Phi\rangle$ is irreducible as an $N$-representation, and does not occur as a summand of $V_{i} \mid N$ for any $i \neq 1$. So the $N_{ \pm}$-representation $V_{1}^{\prime} \stackrel{\text { def }}{=} V_{1}\left\langle\Phi_{ \pm}\right\rangle$is irreducible - since

$$
V_{1}^{\prime} \mid N \cong V_{1}\langle\Phi\rangle \oplus V_{1}\left\langle\Phi_{ \pm} \backslash \Phi\right\rangle
$$

— and $V_{1}^{\prime}$ does not occur as a summand of $V_{i} \mid N_{ \pm}$for any $i \neq 1$. Also, since $V_{1}$ is self-conjugate, the elements of $\Psi$, and hence of $\Phi_{ \pm}$, occur in pairs $\pm \phi$. This shows that $V_{1}^{\prime}=V_{1}\left\langle\Phi_{ \pm}\right\rangle$is invariant under the conjugate linear automorphism $j: V_{1} \rightarrow V_{1}$, and hence that it also has quaternion type. Thus, $V \mid N_{ \pm}$contains with multiplicity one the irreducible summand $V_{1}^{\prime}$ of quaternion type, and this contradicts the assumption that $V \mid N_{ \pm}$is a representation of real type.

It remains to extend this criterium to a result which detects characters by restriction to finite subgroups of $G$. As usual, a finite group is called elementary if it is the product of a $p$-group (for some prime $p$ ) and a cyclic group. A finite group $G$ is called $\mathbb{R}$-elementary if it is elementary, or if it contains a normal cyclic subgroup $C \triangleleft G$ of 2-power index with the property that for any $g \in G$, conjugation by $g$ acts on $C$ via the identity or via $\left(x \mapsto x^{-1}\right)$. 
Proposition 1.5. For any class function $f: G \rightarrow \mathbb{C}, f$ is a character of $G$ if and only if its restriction to any finite elementary subgroup of $G$ is a character; and $f$ is a real character of $G$ if and only if its restriction to each finite $\mathbb{R}$-elementary subgroup of $G$ is a real character.

Proof. When $G$ is finite, the proposition holds by the classical Brauer theorems for detecting characters of finite groups (cf. [Ser, Theorem 21 and Proposition $36]$ ). So it will suffice to show that $f$ is a (real) character of $G$ if and only if its restrictions to all finite subgroups of $G$ are (real) characters. By Proposition 1.2, it suffices to prove this when the connected component $G_{0}$ of $G$ is a torus.

Assume now that $G_{0}=T$ is a torus. We can choose a sequence $H_{1} \subseteq H_{2} \subseteq$ $H_{3} \subseteq \ldots$ of subgroups of $G$ such that each $H_{i}$ intersects all connected components of $G$, and such that the union of the $H_{i}$ is dense in $G$. The simplest way to see this is to set $n=|G / T|$, let ${ }_{n} T \subseteq T$ denote the $n$-torsion subgroup, and note that the homomorphism $H^{2}\left(G / T ;{ }_{n} T\right) \rightarrow H^{2}(G / T ; T)$ is surjective since $n \cdot H^{2}(G / T ; T)=0$. Hence there is a subgroup $H_{0} \subseteq G$ such that $H_{0} \cap T={ }_{n} T$ and $\left\langle H_{0}, T\right\rangle=G$; and we can define $H_{k}=\left\langle H_{0},{ }_{n \cdot 2^{k}} T\right\rangle$ for each $k>0$.

Let $f \in \mathrm{Cl}(G)$ be any class function whose restriction to each $H_{i}$ is a character. For each character $\chi$ of $G$,

$$
\langle f, \chi\rangle_{G} \stackrel{\text { def }}{=} \int_{G} f \cdot \bar{\chi}=\lim _{i \rightarrow \infty}\left(\frac{1}{\left|H_{i}\right|} \sum_{g \in H_{i}} f(g) \cdot \overline{\chi(g)}\right)=\lim _{i \rightarrow \infty}\langle f, \chi\rangle_{H_{i}}
$$

(by definition of the Riemann integral); and $\langle f, \chi\rangle_{H_{i}} \in \mathbb{Z}$ for each $i$ since $f \mid H_{i}$ is a character of $H_{i}$. Thus, $\langle f, \chi\rangle_{G} \in \mathbb{Z}$ for each $\chi$, and so $f$ is a character of $G$ by Lemma 1.3(a). And if $f \mid H_{i}$ is a real character for each $i$, then $f$ is real valued (the union of the $H_{i}$ being dense in $\left.G\right),\langle f, \chi\rangle_{G}=\lim _{i \rightarrow \infty}\langle f, \chi\rangle_{H_{i}} \in 2 \mathbb{Z}$ for each character $\chi$ of quaternion type by Lemma $1.3(\mathrm{~b})$, and so $f$ is a real character by Lemma $1.3(\mathrm{~b})$.

\section{Section 2. Induction for representations of compact Lie groups}

Again, throughout the section, $G$ denotes a fixed compact Lie group. We construct an induction homomorphism $\mathrm{R}(H) \rightarrow \mathrm{R}(G)$, for an arbitrary closed subgroup $H \subseteq G$, by first defining it between the groups of class functions, and then using the results of Section 1 to show that it sends characters to characters.

The following lemma is useful for constructing continuous functions on $G$, and on certain closed subsets of $G$.

Lemma 2.1. Let $\mathcal{F}$ be any set of closed subgroups of $G$, closed under conjugation and closed in the space of all subgroups (with the Hausdorff topology). Set $G_{\mathcal{F}}=\cup_{H \in \mathcal{F}} H$ : the union of the subgroups in $\mathcal{F}$. Then for any function $f: G_{\mathcal{F}} \rightarrow$ 
$\mathbb{C}$ invariant under conjugation, $f$ is continuous on $G_{\mathcal{F}}$ if $f \mid H$ is continuous for all $H \in \mathcal{F}$.

Proof. Fix any conjugation invariant function $f: G_{\mathcal{F}} \rightarrow \mathbb{C}$ such that $f \mid H$ is continuous for all $H \in \mathcal{F}$. It will suffice to show, for any sequence $g_{i} \rightarrow g$ in $G_{\mathcal{F}}$, that some subsequence of the $f\left(g_{i}\right)$ converges to $f(g)$. Since if $f$ is not continuous at $g$, then there is $\epsilon>0$ and a sequence $\left\{g_{i}\right\}$ in $G_{\mathcal{F}}$ converging to $g$ such that $\left|f\left(g_{i}\right)-f(g)\right|>\epsilon$ for all $i$.

Fix such $g_{i}$ and $g$; and for each $i$ choose $H_{i} \in \mathcal{F}$ such that $g_{i} \in H_{i}$. Since $\mathcal{F}$ is closed in the space of closed subgroups of $G$, and since this space is compact (cf. [tD, Proposition IV.3.2(i)]), we can replace the $g_{i}$ by a subsequence and assume that the $H_{i}$ converge to some subgroup $H \in \mathcal{F}$. By [tD, Theorem I.5.9], there exist elements $a_{i} \rightarrow e$ such that $a_{i} H_{i} a_{i}^{-1} \subseteq H$ for $i$ sufficiently large. And hence

$$
\lim _{i \rightarrow \infty} f\left(g_{i}\right)=\lim _{i \rightarrow \infty} f\left(a_{i} g_{i} a_{i}^{-1}\right)=f(g)
$$

since $f \mid H$ is continuous.

The next lemma is also rather technical, and will be used later to show that the induction homomorphism we define for class functions is well defined.

Lemma 2.2. Fix a closed subgroup $H \subseteq G$ and an element $g \in G$, and let $(G / H)^{g}$ be the fixed point set of the action of $g$ on $G / H$. A coset $a H \in G / H$ lies in $(G / H)^{g}$ if and only if $a^{-1} g a \in H$. And if $a_{1} H$ and $a_{2} H$ lie in the same connected component of $(G / H)^{g}$, then $a_{2} H=x a_{1} H$ for some $x \in C_{G}(g)$. In particular, in this situation, $a_{1}^{-1} g a_{1}$ is conjugate in $H$ to $a_{2}^{-1} g a_{2}$.

Proof. For any $a \in G, a H \in(G / H)^{g}$ if and only if $g a H=a H$, if and only if $a^{-1} g a \in H$. Also, if $a_{2} H=x a_{1} H$ for any $a_{1}, a_{2} \in G$ and any $x \in C_{G}(g)$, then $a_{1}^{-1} g a_{1}$ and $a_{2}^{-1} g a_{2}$ are conjugate by an element of $H$.

Now fix an element $a H \in(G / H)^{g}$. Let $C_{G}(g)_{0}$ be the identity connected component of the centralizer of $g$. We must show that the connected component of $a H$ in $(G / H)^{g}$ is $C_{G}(g)_{0} \cdot a H$. Equivalently, via translation by $a^{-1}$, we must show that the connected component of $e H$ in $(G / H)^{a^{-1} g a}$ is $C_{G}\left(a^{-1} g a\right) \cdot e H$. So upon replacing $a^{-1} g a$ by $g$, we are reduced to the case where $a=e$ and $g \in H$.

Let $\mathfrak{h} \subseteq \mathfrak{g}$ denote the Lie algebras of $H \subseteq G$. For all $x \in G, x H \in(G / H)^{g}$ if and only if $x H=g x H=g x g^{-1} H$. In particular, $C_{G}(g) \cdot H \subseteq(G / H)^{g}$; and the tangent plane at $e H$ to the manifold $(G / H)^{g}$ is $(\mathfrak{g} / \mathfrak{h})^{\operatorname{Ad}(g)}$ (the fixed point set of the adjoint action of $g$ on $\mathfrak{g} / \mathfrak{h})$. Also, the projection of $\mathfrak{g}$ onto $\mathfrak{g} / \mathfrak{h}$ is split, equivariantly with respect to the action of the compact group $H$, and so $\mathfrak{g}^{\operatorname{Ad}(g)}$ surjects onto $(\mathfrak{g} / \mathfrak{h})^{\operatorname{Ad}(g)}$. Since $\mathfrak{g}^{\operatorname{Ad}(g)}$ is the Lie algebra of $C_{G}(g)$, this shows that the two submanifolds $C_{G}(g)_{0} \cdot H \subseteq(G / H)^{g}$ have the same dimension, and hence that $C_{G}(g)_{0} \cdot H$ is the connected component of $e H$ in $(G / H)^{g}$. 
We can now define the induction homomorphism for class functions, motivated by the formula given by Segal [Seg, p. 119].

Propositition 2.3. Let $H \subseteq G$ be any closed subgroup. Then there is a homomorphism

$$
\operatorname{Ind}_{H}^{G}: \mathrm{Cl}(H) \longrightarrow \mathrm{Cl}(G)
$$

determined (uniquely) by the following formula. Fix any $g \in G$, let $F_{1}, \ldots, F_{k}$ be the connected components of $(G / H)^{g}$, and choose elements $a_{i} H \in F_{i}$. Then for any $f \in \mathrm{Cl}(H)$,

$$
\operatorname{Ind}_{H}^{G}(f)(g)=\sum_{i=1}^{k} \chi\left(F_{i}\right) \cdot f\left(a_{i}^{-1} g a_{i}\right) .
$$

Proof. Fix any $f \in \mathrm{Cl}(H)$. By Lemma 2.2, for each $g \in G, \operatorname{Ind}_{H}^{G}(f)(g)$ is independent of the choice of representatives $a_{i} H$ for the components of $(G / H)^{g}$. Also, $\operatorname{Ind}_{H}^{G}(f)$ is conjugation invariant by definition; and it only remains to check that it is continuous.

Let $\mathcal{F}$ be the family of abelian subgroups of $G$. Clearly, $\mathcal{F}$ is closed in the Hausdorff topology, and its union is all of $G$. By Lemma 2.1, it will suffice to show that $f \mid A$ is continuous for each $A \in \mathcal{F}$. Let $X$ be a connected component of some subgroup $A \in \mathcal{F}$; we can assume that $X$ generates $\pi_{0}(A)$. For any $g \in X, A /\langle g\rangle$ is connected (where $\langle g\rangle$ is the closure of the subgroup generated by $g$ ); and hence is a torus (or trivial). If $(G / H)^{g}=\coprod_{i=1}^{k} F_{i}$, where the $F_{i}$ are connected components, then $(G / H)^{A}=\coprod_{i=1}^{k}\left(F_{i}\right)^{A /\langle g\rangle}$ and $\chi\left(\left(F_{i}\right)^{A /\langle g\rangle}\right)=\chi\left(F_{i}\right)$ for each $i$. Thus, if we write $(G / H)^{A}=\coprod_{j=1}^{m} E_{j}$ (where the $E_{j}$ are the connected components), and choose elements $b_{j} H \in E_{j}$, then

$$
\operatorname{Ind}_{H}^{G}(f)(g)=\sum_{j=1}^{m} \chi\left(E_{j}\right) \cdot f\left(b_{j}^{-1} g b_{j}\right) .
$$

This formula holds for all $g \in X$, and shows that $\operatorname{Ind}_{H}^{G}(f)$ is continuous on $X$.

The following double coset formula for induction and restriction of class functions is analogous to that shown by Feshbach [Fe] for equivariant cohomology theories. It was shown for representations by Snaith [Sn, Theorem 2.4], using Segal's definition. We prove it here for class functions, using directly the definition in Proposition 2.3.

Lemma 2.4. Fix closed subgroups $H, K \subseteq G$, and write

$$
K \backslash G / H=\coprod_{i=1}^{k} U_{i}
$$


where each $U_{i}$ is a connected component of one orbit type for the action of $K$ on $G / H$. Fix elements $a_{1}, \ldots, a_{k} \in G$ such that $K a_{i} H \in U_{i}$. For each $i$, let $\varphi_{i}: \mathrm{Cl}(H) \rightarrow \mathrm{Cl}(K)$ denote the composite

$$
\varphi_{i}: \mathrm{Cl}(H) \stackrel{\text { Res }}{\longrightarrow} \mathrm{Cl}\left(a_{i}^{-1} K a_{i} \cap H\right) \stackrel{\operatorname{conj}\left(a_{i}^{-1}\right)}{\longrightarrow} \mathrm{Cl}\left(K \cap a_{i} H a_{i}^{-1}\right) \stackrel{\text { Ind }}{\longrightarrow} \mathrm{Cl}(K) .
$$

Then, as functions from $\mathrm{Cl}(H)$ to $\mathrm{Cl}(K)$,

$$
\operatorname{Res}_{K}^{G} \circ \operatorname{Ind}_{H}^{G}=\sum_{i=1}^{k} \chi^{\sharp}\left(U_{i}\right) \cdot \varphi_{i} ;
$$

where for each $i$,

$$
\chi^{\sharp}\left(U_{i}\right)=\chi\left(\overline{U_{i}}, \overline{U_{i}} \backslash U_{i}\right)=\chi\left(\overline{U_{i}}\right)-\chi\left(\overline{U_{i}} \backslash U_{i}\right) .
$$

Proof. Fix elements $f \in \mathrm{Cl}(H)$ and $g \in K$. We will compare the two maps in (1), when evaluated on a given class function $f$ and a given element $g$.

Let $\widetilde{U}_{i} \subseteq G / H$ denote the inverse image of $U_{i}$ under the projection to $K \backslash G / H$. Let $F_{1}, \ldots, F_{m}$ be the connected components of $(G / H)^{g}$. Thus, $G / H=\coprod_{i=1}^{k} \widetilde{U}_{i}$ and $(G / H)^{g}=\coprod_{j=1}^{m} F_{j}$. For each $i, j$, set

$$
V_{i j}=\left(K \cdot a_{i} H\right) \cap F_{j} \subseteq \widetilde{U}_{i} \cap F_{j} \subseteq G / H
$$

(note that $V_{i j}$ need not be connected). Then the $V_{i j} \rightarrow \widetilde{U}_{i} \cap F_{j} \rightarrow U_{i}$ are fibration sequences, and so

$$
\chi\left(F_{j}\right)=\sum_{i=1}^{k} \chi^{\sharp}\left(\widetilde{U}_{i} \cap F_{j}\right)=\sum_{i=1}^{k} \chi\left(V_{i j}\right) \cdot \chi^{\sharp}\left(U_{i}\right) .
$$

for each $j$. Fix elements $b_{i j} \in K$, for each $i, j$, such that $b_{i j} a_{i} H \in V_{i j}$. Then by definition of the induction map (and Lemma 2.1),

$$
\begin{aligned}
\left(\operatorname{Res}_{K}^{G} \circ \operatorname{Ind}_{H}^{G}\right)(f)(g) & =\operatorname{Ind}_{H}^{G}(f)(g)=\sum_{i=1}^{k} \sum_{j=1}^{m} \chi\left(V_{i j}\right) \cdot \chi^{\sharp}\left(U_{i}\right) \cdot f\left(a_{i}^{-1} b_{i j}^{-1} g b_{i j} a_{i}\right) \\
& =\sum_{i=1}^{k} \chi^{\sharp}\left(U_{i}\right) \cdot \sum_{j=1}^{m} \chi\left(V_{i j}\right) \cdot\left(f \circ \operatorname{conj}\left(a_{i}^{-1}\right)\right)\left(b_{i j}^{-1} g b_{i j}\right) .
\end{aligned}
$$

And for each $i$, if we set $K_{i}=K \cap a_{i} H a_{i}^{-1}$ (the isotropy subgroup of the action of $K$ on $\left.a_{i} H \in G / H\right)$, then $\left(K / K_{i}\right)^{g} \cong\left(K \cdot a_{i} H\right)^{g}=\coprod_{j=1}^{m} V_{i j}(\subseteq G / H)$; and so

$$
\sum_{j=1}^{m} \chi\left(V_{i j}\right) \cdot\left(f \circ \operatorname{conj}\left(a_{i}^{-1}\right)\right)\left(b_{i j}^{-1} g b_{i j}\right)=\operatorname{Ind}_{K_{i}}^{K}\left(f \circ \operatorname{conj}\left(a_{i}^{-1}\right)\right)(g)=\varphi_{i}(f)(g) .
$$


When $G$ is finite, the formula given in Proposition 2.3 is just the usual formula for the induction of characters (cf. [Ser, Theorem 12]). Hence by the double coset formula in Lemma 2.4, for each character (real character) $\chi$ of $H$ and each finite subgroup $K \subseteq G,\left(\operatorname{Ind}_{H}^{G}(\chi)\right) \mid K$ is a character (or real character) of $K$. The detection result of Proposition 1.5 now applies to show:

Theorem 2.5. The homomorphism $\operatorname{Ind}_{H}^{G}$ of Proposition 2.3 sends characters to characters, and sends real characters to real characters. It thus restricts to homomorphisms

$$
\operatorname{Ind}_{H}^{G}: \mathrm{R}(H) \longrightarrow \mathrm{R}(G) \quad \text { and } \quad \operatorname{Ind}_{H}^{G}: \mathrm{RO}(H) \longrightarrow \mathrm{RO}(G) .
$$

These induction homomorphisms are in fact functorial; i.e., they compose in the expected way.

Lemma 2.6. For any closed subgroups $K \subseteq H \subseteq G$,

$$
\operatorname{Ind}_{K}^{G}=\operatorname{Ind}_{H}^{G} \circ \operatorname{Ind}_{K}^{H}: \mathrm{Cl}(K) \longrightarrow \mathrm{Cl}(G),
$$

and hence

$$
\operatorname{Ind}_{K}^{G}=\operatorname{Ind}_{H}^{G} \circ \operatorname{Ind}_{K}^{H}: \mathrm{R}(K) \longrightarrow \mathrm{R}(G) .
$$

Proof. Fix any element $g \in G$, and consider the projection $(G / K)^{g} \stackrel{\mathrm{pr}}{\longrightarrow}(G / H)^{g}$. For any $a H \in(G / H)^{g}$,

$$
\operatorname{pr}^{-1}(a H)=\left\{a h K \mid h \in H, h^{-1}\left(a^{-1} g a\right) h \in K\right\}=a \cdot(H / K)^{a^{-1} g a} .
$$

If $a H$ and $a^{\prime} H$ lie in the same connected component of $(G / H)^{g}$, then $a^{\prime} H=x a H$ for some $x \in C_{G}(g)$ (Lemma 2.2), and so $\mathrm{pr}^{-1}\left(a^{\prime} H\right)=x \cdot \mathrm{pr}^{-1}(a H)$. It follows that pr is a fibration (fiber bundle) over each connected component of $(G / H)^{g}$. The result now follows from the definition of the induction homomorphisms (Proposition 2.3), together with the multiplicativity of Euler characteristics in a fibration.

We leave it as an exercise to check that this induction homomorphism is the same as that defined by Segal in [Seg] (use the formula given in [Seg, p. 119]).

It is not hard to prove Frobenius reciprocity for induction and restriction of representations, using the definition given here. And that in turn implies, for example, that the induction map $\operatorname{Ind}_{N(T)}^{G}: \mathrm{R}(N(T)) \rightarrow \mathrm{R}(G)$ is always surjective, and split by the restriction map. See also [Sn, Section 2.3] for the proofs of these results using Segal's definition of induction. 


\section{Section 3. Representations supported by $p$-toral subgroups}

Again, throughout this section, $G$ will be a fixed compact Lie group, and $G_{0}$ will denote its identity connected component. Let $\mathcal{S}_{\mathcal{P}}(G)$ denote the family of $p$-toral subgroups of $G$, for all primes $p$. We now consider the groups

$$
\mathrm{R}_{\mathcal{P}}(G)=\lim _{P \in \mathcal{S}_{\mathcal{P}}(G)} \mathrm{R}(P) \quad \text { and } \quad \mathrm{RO}_{\mathcal{P}}(G)=\lim _{P \in \overleftarrow{\mathcal{S}_{\mathcal{P}}}(G)} \mathrm{RO}(P)
$$

where the limits are taken with respect to inclusion and conjugation; and the natural "restriction" maps

$$
\mathrm{rs}_{G}^{\mathrm{U}}: \mathrm{R}(G) \longrightarrow \mathrm{R}_{\mathcal{P}}(G) \quad \text { and } \quad \mathrm{rs}_{G}^{\mathrm{O}}: \mathrm{RO}(G) \longrightarrow \mathrm{RO}_{\mathcal{P}}(G) .
$$

These groups were shown in [JO] to be naturally isomorphic to the Grothendieck groups $\mathbb{K}(B G)$ and $\mathbb{K} \mathbb{O}(B G)$, respectively, of vector bundles over $B G$ (and rs ${ }_{G}^{\mathrm{U}}$ and $\mathrm{rs}_{G}^{\mathrm{O}}$ are isomorphic to the natural homomorphisms $\mathrm{R}(G) \rightarrow \mathbb{K}(B G)$ and $\mathrm{RO}(G) \rightarrow$ $\mathbb{K} \mathbb{O}(B G))$.

The homomorphisms $\mathrm{rs}_{G}$ are shown here to split as a direct sums of homomorphisms between finitely generated groups, one for each $G / G_{0}$-orbit of irreducible $G_{0}$-representations, and the cokernel of each summand is computed (Theorem 3.9). In particular, this yields necessary and sufficient conditions for $\mathrm{rs}_{G}^{U}$ to be onto (Theorem 3.10 and Corollary 3.11). The orthogonal case seems to be much more complicated; but we do at least show that $\mathrm{rs}_{G}^{O}$ is onto whenever $G$ is finite or $\pi_{0}(G)$ has prime power order (Propositions 3.2 and 3.4), and then give some examples which show that $\mathrm{rs}_{G}^{\mathrm{O}}$ can fail to be onto even when $\mathrm{rs}_{G}^{\mathrm{U}}$ is onto.

It will be useful to define the "character" of an element of $\mathrm{R}_{\mathcal{P}}(G)$. For any compact Lie group $G$, let $G_{\mathcal{P}}$ denote the union of the connected components in $G$ of prime power order in $\pi_{0}(G)$. Let $\mathrm{Cl}\left(G_{\mathcal{P}}\right)$ denote the space of continuous functions $G_{\mathcal{P}} \rightarrow \mathbb{C}$ invariant on conjugacy classes (i.e., the "class functions" on $\left.G_{\mathcal{P}}\right)$.

Lemma 3.1. There is a (unique) character homomorphism

$$
\chi: \mathrm{R}_{\mathcal{P}}(G) \longrightarrow \mathrm{Cl}\left(G_{\mathcal{P}}\right),
$$

such that for any $v=\left(v_{P}\right)_{P \in \mathcal{S}_{\mathcal{P}}(G)} \in \mathrm{R}_{\mathcal{P}}(G), \chi(v) \mid P=\chi_{v_{P}}$ for all $P$ in $\mathcal{S}_{\mathcal{P}}(G)$. Also, $\chi$ sends $\mathrm{R}_{\mathcal{P}}(G)\left(\mathrm{RO}_{\mathcal{P}}(G)\right)$ isomorphically to the subgroup of those class functions on $G_{\mathcal{P}}$ whose restriction to each p-toral subgroup $P \subseteq G$, for all primes $p$, is a character of $P$ (a real character of $P$ ).

Proof. Let $\mathcal{F}$ be the set of $p$-toral subgroups of $G$ (for all primes $p$ ), whose identity connected component is a maximal torus of $G$. Clearly, $\mathcal{F}$ is closed in the Hausdorff topology (note that for $P \in \mathcal{F}$, the order of $\pi_{0}(P)$ is bounded by $\left|N_{G}(T) / T\right|$ ). And by Proposition 1.1, $G_{\mathcal{P}}$ is the union of the $P \in \mathcal{F}$. 
Now, for any $v=\left(v_{P}\right)_{P \in \mathcal{S}_{\mathcal{P}}(G)} \in \mathrm{R}_{\mathcal{P}}(G)$, define $\chi(v): G_{\mathcal{P}} \rightarrow \mathbb{C}$ to be the union of the characters $\chi_{v_{P}}$. This is well defined, and invariant under conjugation, by definition of the inverse limit. Also, $\chi(v)$ is continuous by Lemma 2.1, applied to the family $\mathcal{F}$; and so $\chi(v) \in \mathrm{Cl}\left(G_{\mathcal{P}}\right)$.

The character homomorphism $\chi$ is clearly a monomorphism, and the descriptions of the images of $\mathrm{R}_{\mathcal{P}}(G)$ and $\mathrm{RO}_{\mathcal{P}}(G)$ are immediate from the construction.

We are now ready to study the groups $\mathrm{R}_{\mathcal{P}}(G)$ and $\mathrm{RO}_{\mathcal{P}}(G)$, beginning with the following case.

Proposition 3.2. If $\pi_{0}(G)$ has prime power order, then

$$
r s_{G}^{\mathrm{U}}: \mathrm{R}(G) \stackrel{\cong}{\longrightarrow} \mathrm{R}_{\mathcal{P}}(G) \quad \text { and } \quad r s_{G}^{\mathrm{O}}: \mathrm{RO}(G) \stackrel{\cong}{\longrightarrow} \mathrm{RO}_{\mathcal{P}}(G)
$$

are isomorphisms. Furthermore, for any $G$,

$$
\mathrm{R}_{\mathcal{P}}(G) \cong \lim _{P \in \mathcal{F}_{\mathcal{P}}(G)} \mathrm{R}(P) \quad \text { and } \quad \mathrm{RO}_{\mathcal{P}}(G) \cong \varliminf_{P \in \lim _{\mathcal{\mathcal { F } _ { \mathcal { P } }}}(G)} \mathrm{RO}(P)
$$

where $\mathcal{F}_{\mathcal{P}}(G)$ denotes the family of subgroups $H \subseteq G$ of finite index such that $H / G_{0}$ has prime power order (and the limits are taken with respect to inclusion and conjugation).

Proof. If $\pi_{0}(G)$ is a $p$-group for any prime $p$, then $G=G_{\mathcal{P}}$, and so $\operatorname{rs}_{G}^{\mathrm{U}}$ and $\operatorname{rs}_{G}^{\mathrm{O}}$ are both monomorphisms by Lemma 3.1. To prove that they are isomorphisms, we must show that a class function $f \in \mathrm{Cl}(G)$ is a (real) character if its restriction to all $p$-toral subgroups of $G$ is a (real) character.

Fix a maximal torus $T$ and a Weyl chamber $C \subseteq \mathfrak{t}$, and set $N=N_{G}(T, C)$. Then $N$ is $p$-toral by Proposition 1.1; and by Proposition 1.2 a class function $f \in \mathrm{Cl}(G)$ is a character of $G$ if $f \mid N$ is a character of $N$. This shows that $\operatorname{rs}_{G}^{\mathrm{U}}$ is an isomorphism. If $\pi_{0}(G)$ is a 2-group, then $N_{G}(T, \pm C)$ is 2-toral, and the same argument shows that $\mathrm{rs}_{G}^{O}$ is an isomorphism. Finally, if $\pi_{0}(G)$ is a $p$-group for an odd prime $p$, then for any $v \in \operatorname{RO}_{\mathcal{P}}(G), \chi(v)$ is a real valued character of $G$ whose restriction to $G_{0}$ is a real character of $G_{0}$ (since $\pi_{0}\left(G_{0}\right)=1$ is a 2-group); and so $\chi(v)$ is a real character of $G$ by Lemma 1.3(c).

This finishes the proof of the first statement above. The formulas in (1) now follow immediately (by the transitivity of inverse limits).

The importance of the formulas in (1) above is that they show that the groups $\mathrm{R}_{\mathcal{P}}(G)$ and $\mathrm{RO}_{\mathcal{P}}(G)$, and also the maps $\mathrm{rs}_{G}^{\mathrm{U}}$ and $\mathrm{rs}_{G}^{\mathrm{O}}$, split as sums of groups and maps indexed by the irreducible representations of the identity component $G_{0}$. This will be made more explicit in Theorem 3.9 below. 
The next proposition describes how standard induction techniques apply to study $\mathrm{R}_{\mathcal{P}}(G)$ and $\mathrm{rs}_{G}^{\mathrm{U}}$. Recall that a finite group $\Gamma$ is $p$-elementary if it is a product of a $p$-group and a cyclic group, and is elementary if it is $p$-elementary for some prime $p$. Also, $\Gamma$ is $2-\mathbb{R}$-elementary if it contains a normal cyclic subgroup $C_{m}$ of 2-power index such that any element of $G$ either centralizes $C_{m}$ or acts on it via $\left(a \mapsto a^{-1}\right)$; and is $\mathbb{R}$-elementary if it is elementary or 2 - $\mathbb{R}$-elementary.

Proposition 3.3. (a) For any subgroup $H \subseteq G$ of finite index, there is an induction homomorphism

$$
\operatorname{Ind}_{H}^{G}: \mathrm{R}_{\mathcal{P}}(H) \longrightarrow \mathrm{R}_{\mathcal{P}}(G)
$$

with the property that for any $v \in \mathrm{R}_{\mathcal{P}}(H)$ and any $g \in G_{\mathcal{P}}$,

$$
\chi\left(\operatorname{Ind}_{H}^{G}(v)\right)(g)=\left(\operatorname{Ind}_{H}^{G}(\chi(V))\right)(g)=\sum_{a H \in(G / H)^{g}} \chi(v)\left(a^{-1} g a\right) .
$$

(b) Let $\mathcal{E}(G)$ and $\mathcal{E}_{\mathbb{R}}(G)$ denote the sets of subgroups $E \subseteq G$ of finite index such that $E / G_{0}$ is elementary or $\mathbb{R}$-elementary, respectively. Then restriction induces isomorphisms

$$
\operatorname{Coker}\left(r s_{G}^{\mathrm{U}}\right) \stackrel{\cong}{\longrightarrow} \lim _{E \in \mathcal{E}(G)} \operatorname{Coker}\left(r s_{E}^{\mathrm{U}}\right) \quad \text { and } \quad \operatorname{Coker}\left(r s_{G}^{\mathrm{O}}\right) \stackrel{\cong}{\lim _{E \in \mathcal{E}_{\mathbb{R}}}(G)} \operatorname{Coker}\left(r s_{E}^{\mathrm{O}}\right)
$$

where the limits are taken with respect to inclusion and conjugation in $G$.

Proof. We regard $\operatorname{Ind}_{H}^{G}$ as a homomorphism $\mathrm{Cl}\left(H_{\mathcal{P}}\right) \rightarrow \mathrm{Cl}\left(G_{\mathcal{P}}\right)$, defined via formula (1). Note that this is just the restriction to $G_{\mathcal{P}}$ of the formula given in Proposition 2.3 (though only in the case where $[G: H]<\infty$ ). In particular, the double coset formula of Lemma 2.4 applies in this situation.

(a) Fix any $v \in \mathrm{R}_{\mathcal{P}}(H)$, and let $\chi=\chi(v) \in \mathrm{Cl}\left(H_{\mathcal{P}}\right)$ be its character. We must show that $\operatorname{Ind}_{H}^{G}(\chi)$ is the character of an element of $\mathrm{R}_{\mathcal{P}}(G)$; or equivalently (by Lemma 3.1) that $\operatorname{Ind}_{H}^{G}(\chi) \mid P$ is a character for all $p$-toral subgroups $P \subseteq G$ (for all primes $p$ ). And for any such $P, g P g^{-1} \cap H$ is $p$-toral for each $g \in G$, so $\chi \mid\left(g P g^{-1} \cap H\right)$ is a character, and hence $\operatorname{Ind}_{H}^{G}(\chi) \mid P$ is a character of $P$ by the double coset formula.

(b) Let $\mathcal{F}(G)$ be the class of subgroups of $G$ of finite index. The functor $H \mapsto$ $\mathrm{R}\left(H / G_{0}\right)$ satisfies the double coset formula and Frobenius reciprocity relations for induction and restriction, and hence is a Green ring over $\mathcal{F}(G)$ in the sense of Dress [Dr]. Also, the double coset formula of Lemma 2.4 says that $H \mapsto \mathrm{R}_{\mathcal{P}}(H)$ and $H \mapsto \operatorname{Coker}\left(\mathrm{rs}_{H}^{\mathrm{U}}\right)$ are both Mackey functors over $\mathcal{F}(G)$ (again in the sense of Dress); and both are modules over $\mathrm{R}\left(-/ G_{0}\right)$ satisfying Frobenius reciprocity. Since $\mathrm{R}\left(G / G_{0}\right)$ is generated by induction from the $\mathrm{R}\left(E / G_{0}\right)$ for $E \in \mathcal{E}(G)$ [Ser, $\S 10.5$, Theorem 19], the "fundamental theorem" of Mackey functors and Green rings says that $F(G) \cong \varliminf_{E \in \mathcal{E}(G)}(F(E))$ for any such module over $\mathrm{R}\left(-/ G_{0}\right)$. 
This is shown in [Dr, Propositions 1.1 $1^{\prime}$ and 1.2], and a more direct proof is given in [Ol, Theorem 11.1].

Similarly, $H \mapsto \mathrm{RO}_{\mathcal{P}}(H)$ and $H \mapsto \operatorname{Coker}\left(\mathrm{rs}_{H}^{\mathrm{O}}\right)$ are Mackey functors over $\mathcal{F}(G)$, and modules over $\operatorname{RO}\left(-/ G_{0}\right)$ satisfying Frobenius reciprocity. Since $\operatorname{RO}\left(G / G_{0}\right)$ is generated by induction from the $\operatorname{RO}\left(E / G_{0}\right)$ for $E \in \mathcal{E}_{\mathbb{R}}(G)$ [Ser, $\S 12.6$, Theorem 27], the same argument applies to show that $\operatorname{Coker}\left(\mathrm{rs}_{G}^{\mathrm{O}}\right) \cong \underbrace{}_{E \in \mathcal{E}_{\mathbb{R}}(G)} \operatorname{Coker}\left(\mathrm{rs}_{E}^{\mathrm{O}}\right)$.

In fact, the induction map $\operatorname{Ind}_{H}^{G}: \mathrm{R}_{\mathcal{P}}(H) \rightarrow \mathrm{R}_{\mathcal{P}}(G)$ is defined for any closed subgroup $H \subseteq G$, using the formula for induction of characters in Proposition 2.3. To see this, one must check, for any $f \in \mathrm{Cl}(H)$, that $\operatorname{Ind}_{H}^{G}(f) \mid G_{\mathcal{P}}=0$ if $f \mid H_{\mathcal{P}}=0$. This would be immediate if we knew that $H \cap G_{\mathcal{P}} \subseteq H_{\mathcal{P}}$; but that is not the case in general. The existence of the induction map is thus slightly more tricky than in the case where $[G: H]<\infty$, but is not difficult.

We now turn to the case of finite groups.

Proposition 3.4. If $G$ is finite, then $r s_{G}^{\mathrm{U}}$ and $r s_{G}^{\mathrm{O}}$ are both surjective.

Proof. By Proposition 3.3(b), it suffices to show that $\mathrm{rs}_{G}^{\mathrm{U}}$ is onto when $G$ is elementary, and that $\mathrm{rs}_{G}^{\mathrm{O}}$ is onto when $G$ is $\mathbb{R}$-elementary. We do this in the orthogonal case only; the unitary case is similar (but simpler).

Assume that $G$ is $\mathbb{R}$-elementary, and fix an element $v=\left(v_{P}\right)_{P \in \mathcal{S}_{\mathcal{P}}(G)} \in$ $\mathrm{RO}_{\mathcal{P}}(G)$. In other words, $v_{P} \in \mathrm{RO}(P)$ for each $p$-subgroup $P \subseteq G$ (for each prime $p|| G \mid)$; and by subtracting a constant character we can assume that $\chi_{v_{P}}(1)=0$ for each $P$. For each $p|| G \mid$, write $v_{p}=v_{\operatorname{Syl}_{p}(G)} \in \operatorname{RO}\left(\operatorname{Syl}_{p}(G)\right)$. It will suffice to show that each $v_{p}$ extends to an element $v_{p}^{\prime} \in \mathrm{R}(G)$ whose character vanishes on all elements of order prime to $p$ (then $v=\operatorname{rs}_{G}^{O}\left(\sum v_{p}^{\prime}\right)$ ). This is clear if $\operatorname{Syl}_{p}(G)$ has a normal complement, since in that case $v_{p}^{\prime}$ can be taken to be the composite of $v_{p}$ with a surjection $G \rightarrow \operatorname{Syl}_{p}(G)$.

The only case left to consider is that where $p$ is odd, $G$ is $2-\mathbb{R}$-elementary, and $\operatorname{Syl}_{p}(G)$ has no normal complement. Set $p^{k}=\left|\operatorname{Syl}_{p}(G)\right|$; then there is a surjection $G \rightarrow D\left(2 p^{k}\right)$, where $D\left(2 p^{k}\right)$ is dihedral of order $2 p^{k}$. One easily checks that any $v_{p} \in \operatorname{RO}\left(C_{p^{k}}\right)$ such that $\chi_{v_{p}}(1)=0$ extends to an element $v_{p}^{\prime \prime} \in \operatorname{RO}\left(D\left(2 p^{k}\right)\right)$ such that $\chi_{v_{p}^{\prime \prime}}(g)=0$ for all $g$ of order prime to $p$. And hence if $v_{p}^{\prime} \in \operatorname{RO}(G)$ is the composite of $v_{p}^{\prime \prime}$ with the surjection $G \rightarrow D\left(2 p^{k}\right)$, then $v_{p}^{\prime} \mid \operatorname{Syl}_{p}(G)=v_{p}$ and $\chi_{v_{p}^{\prime}}$ vanishes on all elements of order prime to $p$.

Recall that for any torus $T$, we let $\mathfrak{t}$ denote the Lie algebra of $T$, and regard the group $T^{*}=\operatorname{Hom}\left(T, S^{1}\right)$ of irreducible characters of $T$ as a lattice in $\mathfrak{t}^{*}=$ $\operatorname{Hom}(\mathfrak{t}, \mathbb{R})$. The following definitions establish some of the notation which will be used when dealing with irreducible characters and representations of groups with torus identity component. 
Definition 3.5. If $G$ is a compact Lie group with identity component $T$, then the support of a $G$-representation $V$ is the $\left(G / T\right.$-invariant) subset $\operatorname{Supp}(V) \subseteq T^{*}$ of all characters of irreducible summands of $V \mid T$. More generally, for any $v \in \mathrm{R}(G)$, $\operatorname{Supp}(v) \in T^{*}$ is the union of the supports of the irreducible $G$-representations which occur in the decomposition of $v$. For any $G / T$-invariant subset $\Phi \subseteq T^{*}$, $\operatorname{Irr}(G, \Phi)$ denotes the set of irreducible $G$-representations with support in $\Phi$, and $\mathrm{R}(G, \Phi) \subseteq \mathrm{R}(G)$ denotes the subgroup of elements with support in $\Phi$. For $\phi \in$ $T^{*}$, we write $(\phi)$ for the $G / T$-orbit of $\phi$ (and write $\operatorname{Irr}(G, \phi)$, etc., if $\phi$ is $G / T$ invariant). Finally, if $V$ is any $G$-representation, then $V\langle\Phi\rangle$ and $V\langle\phi\rangle$ denote the largest summands of $V$ with support in $\Phi$ or $\phi$, respectively.

The descriptions of Coker( $\left.\mathrm{rs}_{G}^{\mathrm{U}}\right)$ in Lemma 3.8 and Theorem 3.9 below will be given in terms of a certain function $\delta(G)$, defined for compact Lie groups whose identity component is a torus and central.

Definition 3.6. Assume that $G$ lies in a central extension $1 \rightarrow T \rightarrow G \rightarrow \Gamma \rightarrow 1$, where $T$ is a torus and $\Gamma$ is a finite group. For each $\phi \in T^{*}$, define

$$
\delta(G, \phi)=\operatorname{gcd}\{\operatorname{dim}(V) \mid V \in \operatorname{Irr}(G, \phi)\} ;
$$

and set

$$
\delta(G)=\operatorname{lcm}\left\{\delta(G, \phi) \mid \phi \in T^{*}\right\} .
$$

The next lemma gives a partial description of this function, independantly of representations; and also lists some of its more technical properties which will be needed in later proofs.

Lemma 3.7. Assume that $G_{0} \subseteq Z(G)$; i.e., that $G$ lies in a central extension $1 \rightarrow T \rightarrow G \rightarrow \Gamma \rightarrow 1$, where $T$ is a torus and $\Gamma$ is finite. Set $e=\operatorname{expt}(T \cap[G, G])$. For each prime $p|| \Gamma \mid$, let $G_{p}$ be a maximal p-toral subgroup of $G$ : the extension of $T$ by a Sylow p-subgroup of $\Gamma$. Then

(a) $\delta(G)=1$ if and only if $e=1$, if and only if $G \cong T \times \Gamma$

(b) $e \mid \delta(G)$ and $\delta(G)^{2}|| \Gamma \mid$

(c) $\delta(G)=\prod_{p|| \Gamma \mid} \delta\left(G_{p}\right)$, and $\delta(G, \phi)=\prod_{p|| \Gamma \mid} \delta\left(G_{p}, \phi\right)$ for all $\phi \in T^{*}$

(d) $\delta\left(G, \phi^{\prime}\right)=\delta(G, \phi)$ for all $\phi^{\prime}, \phi \in T^{*}$ with $\phi^{\prime} \equiv \phi(\bmod e)$

(e) $\delta(G, n \phi)=\delta(G, \phi)$ for all $\phi \in T^{*}$, and all $n \in \mathbb{Z}$ with $(n, e)=1$.

Proof. Note first that for any $H \subseteq G$ of finite index, and any $\phi \in T^{*}$,

$$
\delta(H, \phi)|\delta(G, \phi)|[G: H] \cdot \delta(H, \phi) .
$$

The first relation holds since each $G$-representation with support in $\phi$ can be regarded as an $H$-representation; and the second since $\operatorname{Ind}_{H}^{G}(V)$ has support in $\phi$ for any $H$-representation $V$ with support in $\phi$. 
(b) Fix any $\phi \in T^{*}$, and choose $a \in T \cap[G, G]$ such that $\phi(a)$ generates $\phi(T \cap$ $[G, G])$. Then for any $G$-representation $V$ with support in $\phi, a$ acts on $V$ via multiplication by $\phi(a)$; and since $a \in[G, G], \phi(a) \cdot \operatorname{Id}_{V}$ has determinant $\phi(a)^{\operatorname{dim}(V)}=1$. Thus, $|\phi(a)| \mid \operatorname{dim}(V)$ for all such $V$, and so

$$
|\phi(a)|=|\phi(T \cap[G, G])| \mid \delta(G, \phi) .
$$

In particular, $e=\operatorname{expt}(T \cap[G, G])$ divides $\delta(G)$.

Now fix any $\phi \in T^{*}$, and let $V_{\phi}$ be the 1-dimensional irreducible $T$-representation with character $\phi$. Let $V_{1}, \ldots, V_{k}$ be the irreducible $G$-representations with support in $\phi$. For each $i$, the multiplicity of $V_{i}$ in $\operatorname{Ind}_{T}^{G}\left(V_{\phi}\right)$ is

$$
\operatorname{dim}_{\mathbb{C}}\left(\operatorname{Hom}_{G}\left(\operatorname{Ind}_{T}^{G}\left(V_{\phi}\right), V_{i}\right)\right)=\operatorname{dim}_{\mathbb{C}}\left(\operatorname{Hom}_{T}\left(V_{\phi}, V_{i}\right)\right)=\operatorname{dim}_{\mathbb{C}} V_{i} .
$$

Thus, $|\Gamma|=\operatorname{dim}\left(\operatorname{Ind}_{T}^{G}\left(V_{\phi}\right)\right)=\sum_{i=1}^{k} \operatorname{dim}\left(V_{i}\right)^{2}$. And so $\delta(G, \phi)$, the greatest common divisor of the $\operatorname{dim}\left(V_{i}\right)$, is such that $\delta(G, \phi)^{2}|| \Gamma \mid$.

(a) We prove here the slightly more general equivalence that

$$
\delta(G, \phi)=1 \Longleftrightarrow \phi(T \cap[G, G])=1 \Longleftrightarrow G / \operatorname{Ker}(\phi) \cong T / \operatorname{Ker}(\phi) \times \Gamma .
$$

The third statement clearly implies the first, and the first implies the second by (2).

By the universal coefficient theorem, $H^{2}(\Gamma ; T) \cong \operatorname{Hom}\left(H_{2}(\Gamma), T\right)$; and $T \cap$ $[G, G]$ is the image of the homomorphism $\eta_{G}: H_{2}(\Gamma) \rightarrow T$ which corresponds to $[G]$ as an element of $H^{2}(\Gamma ; T)$. So $G \cong T \times \Gamma$ if $T \cap[G, G]=1$, and $G / \operatorname{Ker}(\phi) \cong$ $T / \operatorname{Ker}(\phi) \times \Gamma$ if $\phi(T \cap[G, G])=1$.

(c) This formula follows immediately from (1), and the fact that $\delta\left(G_{p}, \phi\right)|| G_{p} / T \mid$ is a power of $p$ for each $p$.

(d) If $\phi \equiv 0(\bmod e)$, then $\phi(T \cap[G, G])=1$, and so $\delta(G, \phi)=1$ by (3). If $\phi^{\prime} \equiv \phi \not \equiv 0(\bmod e)$, then the two composites

$$
H_{2}(\Gamma) \stackrel{\eta_{G}}{\longrightarrow} T \underset{\phi^{\prime}}{\stackrel{\phi}{\longrightarrow}} S^{1}
$$

are equal. Hence $(G / \operatorname{Ker}(\phi), \phi) \cong\left(G / \operatorname{Ker}\left(\phi^{\prime}\right), \phi^{\prime}\right)$ as pairs, and $\delta(G, \phi)=\delta\left(G, \phi^{\prime}\right)$. (e) For any $n \in \mathbb{Z}$ and any $G$-representation $V$ with support $\phi, \psi^{n}(V)$ is a virtual representation with support $n \phi$ : since $\chi_{\psi^{n} V}(g t)=\chi_{V}\left(g^{n} t^{n}\right)=\chi_{\psi^{n} V}(g) \cdot \phi(t)^{n}$ for any $g \in G$ and $t \in T$. Cf. [Ad, Lemma 3.61] for details. Also, $V$ and $\psi^{n}(V)$ have the same (virtual) dimension, and hence $\delta(G, n \phi) \mid \delta(G, \phi)$. So by (d), $\delta(G, n \phi)=$ $\delta(G, \phi)$ if $n$ is invertible $\bmod e$.

Ian Leary has pointed out to me that $\delta(G)$ is the greatest common divisor of the indices $[G: H]$ of those subgroups $H \subseteq G$ of finite index such that $H$ splits as a product $H \cong T \times(H / T)$. 
Whenever $G_{0}=T$ is a torus, $\mathrm{R}(G)$ splits as the direct sum, taken over all $G / T$ orbits $(\phi) \subseteq T^{*}$, of the subgroups $\mathrm{R}(G,(\phi))$ of finite rank. In a similar fashion, $\mathrm{rs}_{G}^{\mathrm{U}}$ splits as the direct sum over all $(\phi) \subseteq T^{*}$ of homomorphisms

$$
\mathrm{rs}_{G,(\phi)}: \mathrm{R}(G,(\phi)) \longrightarrow \mathrm{R}_{\mathcal{P}}(G,(\phi)) .
$$

We are now ready to describe the cokernel of each of these summands for such $G$. The key case to consider is that when $T=G_{0}$ is central and $\phi$ is faithful.

Lemma 3.8. Assume that $G$ lies in a central extension $1 \rightarrow T \rightarrow G \stackrel{\sigma}{\rightarrow} \Gamma \rightarrow 1$, where $T \cong S^{1}$, and where $\Gamma$ is finite. Fix a faithful (injective) character $\phi \in T^{*}$. Let $S$ be the set of all conjugacy classes of elements $g \in \Gamma$ such that no two elements in $\sigma^{-1} g$ are conjugate; and let $S_{\mathcal{P}} \subseteq S$ be the set of conjugacy classes of elements of prime power order. For each $g \in S_{\mathcal{P}}$, let $\eta(g)$ be the largest divisor of $\delta\left(C_{G}(g), \phi\right)$ which is prime to the order of $g$. Then

$$
\mathrm{R}(G, \phi) \cong \mathbb{Z}^{|S|}, \quad \mathrm{R}_{\mathcal{P}}(G, \phi) \cong \mathbb{Z}^{\left|S_{\mathcal{P}}\right|}, \quad \text { and } \quad \operatorname{Coker}\left(r s_{G, \phi}\right) \cong \bigoplus_{1 \neq g \in S_{\mathcal{P}}} \mathbb{Z} / \eta(g) .
$$

Proof. A character $\chi$ of $G$ has support in $\phi$ if and only if it satisfies the relation $\chi(g t)=\chi(g) \phi(t)$ for all $g \in G$ and $t \in T$. In particular, since $\phi$ is injective, $\chi(g)=0$ for any $g$ which is conjugate to $g t$ for some $1 \neq t \in T$. Thus, $\operatorname{Cl}(G, \phi)$ is a complex vector space of dimension $|S|$; and by the Peter-Weyl theorem (and the independence of irreducible characters) $\mathrm{R}(G, \phi)$ is a free abelian group of rank $|S|$. Also, $\mathrm{R}_{\mathcal{P}}(G, \phi)$ is torsion free (it is detected by characters defined on $G_{\mathcal{P}}$ ), and $\operatorname{Ker}\left(\operatorname{rs}_{G, \phi}\right)$ is the set of elements of $\mathrm{R}(G, \phi)$ whose characters vanish on $G_{\mathcal{P}}$. So the image of $\operatorname{rs}_{G, \phi}$ is free of rank $\left|S_{\mathcal{P}}\right|$; and once we have shown that $\mathrm{rs}_{G, \phi}$ has finite cokernel it will follow that $\mathrm{R}_{\mathcal{P}}(G, \phi)$ is a free abelian group of the same rank.

The computation of the cokernel of $\mathrm{rs}_{G, \phi}$ will be carried out in two steps.

Step 1. Assume first that $\Gamma$ is $p$-elementary for some prime $p$. Then we can write $G=C_{n} \times P$, where $C_{n}$ is cyclic of order $n$ prime to $p$, and where $P$ is $p$-toral. In particular, $\mathrm{R}(G) \cong \mathrm{R}\left(C_{n}\right) \otimes \mathrm{R}(P)$ and $\mathrm{R}(G, \phi) \cong \mathrm{R}\left(C_{n}\right) \otimes \mathrm{R}(P, \phi)$. Let $\mathrm{IR}(-)$ denote the augmentation ideal of $\mathrm{R}(-)$, and similarly for $\operatorname{IR}_{\mathcal{P}}(-)$. Consider the following commutative diagram with split short exact rows:

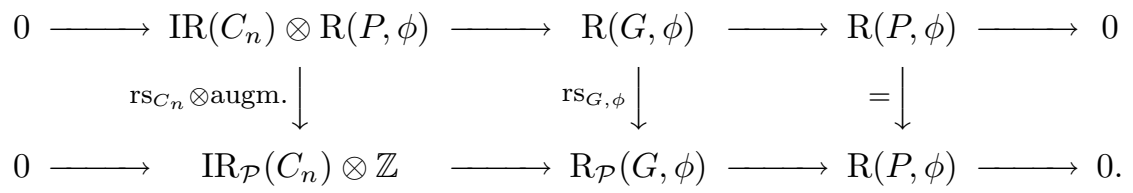

Here, $\operatorname{IR}_{\mathcal{P}}\left(C_{n}\right)$ is the product of the $\operatorname{IR}\left(\operatorname{Syl}_{q}\left(C_{n}\right)\right)$ for $q \mid n$, and any $v \in \operatorname{IR}\left(\operatorname{Syl}_{q}\left(C_{n}\right)\right)$ lifts to an element of $\operatorname{IR}\left(C_{n}\right)$ whose character vanishes on other Sylow subgroups. Hence $\operatorname{IR}\left(C_{n}\right)$ surjects onto $\operatorname{IR}_{\mathcal{P}}\left(C_{n}\right)$, and so

$$
\operatorname{Coker}\left(\operatorname{rs}_{G, \phi}\right) \cong \operatorname{Coker}\left(\operatorname{rs}_{C_{n}} \otimes \operatorname{augm} .\right) \cong \operatorname{IR}_{\mathcal{P}}\left(C_{n}\right) \otimes \operatorname{Coker}[\mathrm{R}(P, \phi) \stackrel{\text { augm. }}{\longrightarrow} \mathbb{Z}] \text {. }
$$


The cokernel of this augmentation map is by definition $\mathbb{Z} / \delta(P, \phi)$, and so

$$
\operatorname{Coker}\left(\mathrm{rs}_{G, \phi}\right) \cong \operatorname{IR}_{\mathcal{P}}\left(C_{n}\right) \otimes(\mathbb{Z} / \delta(P, \phi)) .
$$

Step 2. Now assume that $G$ is arbitrary. Let $\mathcal{E}(G)$ be the set of subgroups of $G$ of finite index such that $E / T$ is elementary, and (for each prime $p|| \Gamma \mid$ ) let $\mathcal{E}_{p}(G)$ be the set of those $E \in \mathcal{E}(G)$ such that $E / T$ is $p$-elementary. By Proposition 3.3, $\operatorname{Coker}\left(\mathrm{rs}_{G, \phi}\right)$ is the inverse limit of the groups $\operatorname{Coker}\left(\mathrm{rs}_{E, \phi}\right)$, taken over all $E \in \mathcal{E}(G)$. By (1), $\operatorname{Coker}\left(\mathrm{rs}_{E, \phi}\right)$ is a finite $p$-group for all $E \in \mathcal{E}_{p}(G)$. Hence $\operatorname{Coker}\left(\operatorname{rs}_{G, \phi}\right)$ is finite; and (for each $\left.p\right) \operatorname{Coker}\left(\operatorname{rs}_{G, \phi}\right)_{(p)}$ is the inverse limit of the $\operatorname{Coker}\left(\mathrm{rs}_{E, \phi}\right)$ for $E \in \mathcal{E}_{p}(G)$.

Fix a prime $p|| \Gamma \mid$; we want to determine the $p$-power torsion in $\operatorname{Coker}\left(\mathrm{rs}_{G, \phi}\right)$. If $K^{\prime} \subseteq K$ are finite cyclic subgroups of order prime to $p$, then the composite

$$
\operatorname{IR}\left(K^{\prime}\right)_{(p)} \stackrel{\text { Ind }}{\longrightarrow} \operatorname{IR}(K)_{(p)} \stackrel{\text { Res }}{\longrightarrow} \operatorname{IR}\left(K^{\prime}\right)_{(p)}
$$

is multiplication by $\left[K: K^{\prime}\right]$, and hence an isomorphism. Thus, if $K$ is cyclic of order prime to $p$, we can split

$$
\operatorname{IR}_{\mathcal{P}}(K)_{(p)}=\bigoplus_{q|| K \mid} \operatorname{IR}\left(\operatorname{Syl}_{q}(K)\right) \cong \bigoplus_{1 \neq K^{\prime} \subseteq K_{\mathcal{P}}} \widetilde{\operatorname{IR}}\left(K^{\prime}\right)_{(p)}
$$

(i.e., taking the second sum over subgroups of prime power order). Here, $\widetilde{\operatorname{IR}}\left(K^{\prime}\right) \subseteq$ $\operatorname{IR}\left(K^{\prime}\right)$ is the kernel of the map given by restriction to the subgroup of prime index, and is free with rank equal to the number of generators of $K^{\prime}$.

For each $n|| \Gamma \mid$ prime to $p$, let $\mathrm{Cyc}_{n}$ be the set of all cyclic subgroups $K \subseteq \Gamma$ of order $n$ if $n$ is a prime power, and set $\mathrm{Cyc}_{n}=\emptyset$ otherwise. By Lemma 3.7(c), for any maximal $p$-toral subgroup $P \subseteq H, \delta(P, \phi)$ is the largest power of $p$ dividing $\delta(H, \phi)$. So with the help of (1) we now get

$$
\begin{aligned}
& \operatorname{Coker}\left(\mathrm{rs}_{G, \phi}\right)_{(p)} \cong \varliminf_{E \in \lim _{p}(G)} \operatorname{Coker}\left(\mathrm{rs}_{E, \phi}\right) \\
& \cong \bigoplus_{p \nmid n|| \Gamma \mid}\left(\lim _{K \in \mathrm{Cyc}_{n}}\left(\widetilde{\operatorname{IR}}(K) \otimes \mathbb{Z} / \delta\left(\sigma^{-1}\left(C_{\Gamma}(K)\right), \phi\right)\right)_{(p)}\right)
\end{aligned}
$$

For each $n=q^{k}$ (where $q \neq p$ is prime), set

$$
\mathrm{Cyc}_{n}^{\prime}=\left\{K=\langle g\rangle \in \mathrm{Cyc}_{n} \mid \text { no two elts. in } \sigma^{-1} g \text { conjugate in } G\right\} .
$$

Fix some $K \in \mathrm{Cyc}_{q^{k}} \backslash \mathrm{Cyc}_{q^{k}}^{\prime}$, and let $K^{\prime} \subseteq K$ be the subgroup of index $q$. Then there exists $x \in N_{G}\left(\sigma^{-1} K\right)$ such that for each $g \in \sigma^{-1}\left(K \backslash K^{\prime}\right), x g x^{-1}=g t$ for some $1 \neq t \in T$. The character of any element $v \in \widetilde{\mathrm{IR}}(K) \cong \widetilde{\mathrm{IR}}\left(\sigma^{-1} K, \phi\right)$ vanishes 
on $\sigma^{-1} K^{\prime}$; and hence (since $\left.\chi_{v}(g t)=\chi_{v}(g) \cdot \phi(t)\right) v$ is fixed by the action of $x$ only if $v=0$. Thus, $x$ acts on $\widetilde{\mathrm{IR}}(K)$ with trivial fixed point set; and in particular such terms contribute nothing to the limit in (3).

Formula (3) thus reduces to a sum, over conjugacy class representatives for all $K \in \mathrm{Cyc}_{n}^{\prime}$, of the groups

$$
H^{0}\left(N_{G}\left(\sigma^{-1} K\right) ; \widetilde{\mathrm{IR}}_{\mathcal{P}}(K)\right) \otimes\left(\mathbb{Z} / \delta\left(C_{G}\left(\sigma^{-1} K\right), \phi\right)\right)_{(p)} .
$$

The first factor here is free of rank equal to the number of $\Gamma$-conjugacy classes of generators of $K$. The formula for $\operatorname{Coker}\left(\mathrm{rs}_{G, \phi}\right)$ now follows upon taking the product over all primes $p|| \Gamma \mid$.

As an example, consider the group $G=C_{n} \times\left(S^{1} \times{ }_{C_{2}} Q(8)\right)$, where $n$ is odd, $Q(8)$ is a quaternion group of order 8 , and the second product is taken while identifying the central elements of order 2 in $S^{1}$ and $Q(8)$. By Lemma 3.8, if $\phi \in T^{*}$ is a generator, then $\operatorname{rs}_{G, k \phi}$ is onto for $k$ even, while $\operatorname{Coker}\left(\mathrm{rs}_{G, k \phi}\right) \cong \mathbb{Z} / 2 \otimes \operatorname{IR}_{\mathcal{P}}\left(C_{n}\right) \neq 0$ if $k$ is odd.

The groups dealt with in Lemma 3.8 seem quite specialized, but we are now ready to show that the general case - for an arbitrary compact Lie group $G$ can always be reduced to the cases handled there.

Theorem 3.9. Let $G$ be any compact Lie group. Fix a maximal torus $T \subseteq G$ and a Weyl chamber $C \subseteq \mathfrak{t}$, and set $N=N(T, C) \subseteq G$. Then $r s_{G}^{U}$ splits as a direct sum of homomorphisms

$$
r s_{G,\left(V_{0}\right)}: \mathrm{R}\left(G,\left(V_{0}\right)\right) \longrightarrow \mathrm{R}_{\mathcal{P}}\left(G,\left(V_{0}\right)\right)
$$

taken over all $G / G_{0}$-orbits $\left(V_{0}\right) \subseteq \operatorname{Irr}\left(G_{0}\right)$.

For any $V_{0} \in \operatorname{Irr}\left(G_{0}\right)$, let $\phi$ be the maximal weight of $V_{0}$ in $C^{*}$, let $N_{\phi} \subseteq N$ be the subgroup of elements which fix $\phi$, and set $K_{\phi}=\operatorname{Ker}(\phi) \subseteq T$. Then the assignment $([V] \mapsto[V\langle\phi\rangle])$ induces isomorphisms

$$
\mathrm{R}\left(G,\left(V_{0}\right)\right) \stackrel{\cong}{\longrightarrow}\left(N_{\phi} / K_{\phi}, \phi\right), \quad \mathrm{R}_{\mathcal{P}}\left(G,\left(V_{0}\right)\right) \stackrel{\cong}{\longrightarrow} \mathrm{R}_{\mathcal{P}}\left(N_{\phi} / K_{\phi}, \phi\right),
$$

and

$$
\operatorname{Coker}\left(r s_{G,\left(V_{0}\right)}\right) \stackrel{\cong}{\longrightarrow} \operatorname{Coker}\left(r s_{N_{\phi} / K_{\phi}, \phi}\right)
$$

Proof. By Lemma 3.2, $\mathrm{R}_{\mathcal{P}}(G)$ is the inverse limit of the representation rings $\mathrm{R}(H)$, taken over all $H \subseteq G$ of finite index such that $H / G_{0}$ has prime power order. Since each $\mathrm{R}(H)$ splits as a sum of finitely generated groups indexed by the $G / G_{0}$-orbits $\left(V_{0}\right) \in \operatorname{Irr}\left(G_{0}\right)$, we now see that $\mathrm{R}_{\mathcal{P}}(G)$ also splits as such a sum. And hence $\operatorname{rs}_{G}^{\mathrm{U}}$ also splits as a direct sum of homomorphisms $\operatorname{rs}_{G,\left(V_{0}\right)}$. 
Now fix $V_{0} \in \operatorname{Irr}\left(G_{0}\right)$ and let $\phi$ be its maximal weight in $C^{*}$. Write $\Phi=(\phi)$ for short: the $N / T$-orbit of $\phi \in C_{T}^{*}$. By Proposition 1.4, the assignment $[V] \mapsto$ $[V\langle\Phi\rangle]$ defines a bijection from $\operatorname{Irr}\left(G,\left(V_{0}\right)\right)$ to $\operatorname{Irr}(N, \Phi)$, and hence an isomorphism $\mathrm{R}\left(G,\left(V_{0}\right)\right) \stackrel{\cong}{\longrightarrow} \mathrm{R}(N, \Phi)$. Similarly, it induces isomorphisms $\mathrm{R}\left(H,\left(V_{0}\right)\right) \stackrel{\cong}{\longrightarrow}$ $\mathrm{R}(H \cap N, \Phi)$ for each $H \subseteq G$ of finite index, and upon taking the inverse limit over all such $H$ for which $H / G_{0}$ has prime power order we get an isomorphism $\mathrm{R}_{\mathcal{P}}\left(G,\left(V_{0}\right)\right) \stackrel{\cong}{\longrightarrow} \mathrm{R}_{\mathcal{P}}(N, \Phi)$. And this in turn induces an isomorphism between the cokernels of $\mathrm{rs}_{G,\left(V_{0}\right)}$ and $\mathrm{rs}_{N, \Phi}$.

The homomorphism $\mathrm{R}(N, \Phi) \rightarrow \mathrm{R}\left(N_{\phi}, \phi\right) \cong \mathrm{R}\left(N_{\phi} / K_{\phi}, \phi\right)$, defined by sending $[V]$ to $[V\langle\phi\rangle]$, is an isomorphism: its inverse is the induction map $[V] \mapsto$ $\left[\operatorname{Ind}_{N_{\phi}}^{N}(V)\right]$. This same assignment also defines an isomorphism $\mathrm{R}_{\mathcal{P}}(N, \Phi) \stackrel{\cong}{\longrightarrow}$ $\mathrm{R}_{\mathcal{P}}\left(N_{\phi} / K_{\phi}, \phi\right)$ (whose inverse is again the induction map); and hence defines an isomorphism between the cokernels of $\mathrm{rs}_{N, \Phi}$ and $\mathrm{rs}_{N_{\phi} / K_{\phi}, \phi}$.

The above general description of $\operatorname{Coker}\left(\mathrm{rs}_{G}^{U}\right)$ is rather complicated. In contrast, the conditions for the $\operatorname{map} \operatorname{rs}_{G}^{U}$ to be onto can be formulated more simply.

Theorem 3.10. Let $G$ be any compact Lie group. Fix a maximal torus $T \subseteq G$ and a Weyl chamber $C \subseteq \mathfrak{t}$, and set $N=N(T, C) \subseteq G$. Let $\mathcal{E}^{\prime}(N)$ denote the set of subgroups $E \subseteq N$ of finite index such that $E / T$ is elementary but not of prime power order. Then

$$
\operatorname{expt}\left(\operatorname{Coker}\left(r s_{G}^{U}\right)\right)=\operatorname{lcm}\left\{\delta(E /[E, T]) \mid E \in \mathcal{E}^{\prime}(N)\right\} .
$$

In particular, $r s_{G}^{\mathrm{U}}$ is surjective if and only if $r s_{N}^{\mathrm{U}}$ is surjective, if and only if $T \cap$ $[E, E]=[E, T]$ for all $E \in \mathcal{E}^{\prime}(N)$.

Proof. It is clear from part (c) that the exponent of Coker( $\left(\mathrm{rs}_{G}^{U}\right)$ divides the number given in (1). To show that these are equal, fix any prime $p$, and choose $E \subseteq N$ of finite index such that $E / T$ is $p$-elementary but not a $p$-group. We must show that $\delta(E /[E, T]) \mid \operatorname{expt}\left(\operatorname{Coker}\left(\mathrm{rs}_{G}^{\mathrm{U}}\right)\right)$. Choose any $\phi^{\prime} \in(T /[E, T])^{*} \subseteq T^{*}$ such that $\delta\left(E /[E, T], \phi^{\prime}\right)=\delta(E /[E, T])$. Since $N / T$ acts linearly on $\mathfrak{t}^{*}$ and leaves $C^{*}$ invariant, the fixed set $\left(C^{*}\right)^{E}$ is a cone shaped subspace of $\left(\mathfrak{t}^{*}\right)^{E}$ with nonempty interior. Hence, we can choose $\phi \in C^{*} \cap(T /[E, T])^{*}=\left(C_{T}^{*}\right)^{E}$ such that $\phi \equiv \phi^{\prime}$ modulo the exponent of $\frac{T \cap[E, E]}{[E, T]}$. If $q \neq p$ is any other prime dividing $|E / T|$, then

$$
\delta(E /[E, T], q \phi)=\delta(E /[E, T], \phi)=\delta\left(E /[E, T], \phi^{\prime}\right)=\delta(E /[E, T])
$$

by Lemma 3.7(d,e). And finally, if $g T \in E / T$ is the element of order $q$, then $g T \in S$ in the notation of Lemma 3.8: no two elements in $g T / \operatorname{Ker}(q \phi)$ are conjugate. Thus,

$$
\delta(E /[E, T])=\delta(E /[E, T], q \phi)\left|\operatorname{expt}\left(\operatorname{Coker}\left(\mathrm{rs}_{E, q \phi}\right)\right)\right| \operatorname{expt}\left(\operatorname{Coker}\left(\mathrm{rs}_{G}^{\mathrm{U}}\right)\right)
$$


by Lemma 3.8; and this finishes the proof of formula (1). The necessary and sufficient conditions for $\mathrm{rs}_{G}^{U}$ to be surjective now follow from Lemma 3.7(a).

Since the general condition for $\operatorname{rs}_{G}^{U}$ to be surjective is still somewhat complicated, we now list some special cases which are simpler to formulate.

Corollary 3.11. For any compact Lie group $G, \operatorname{Coker}\left(r s_{G}^{U}\right)$ has finite exponent, and

$$
\operatorname{expt}\left(\operatorname{Coker}\left(r s_{G}^{U}\right)\right)^{2}|| \pi_{0}(G) \mid \text {. }
$$

Furthermore, $r s_{G}^{U}$ is surjective if $G$ satisfies any of the following conditions:

(a) $G$ is finite or connected.

(b) All elements of $\pi_{0}(G)$ have prime power order.

(c) $\pi_{0}(G)$ is a periodic group: all of its Sylow subgroups are cyclic or quaternion.

(d) $Z\left(G_{0}\right)=1$.

(e) $G$ is a semidirect product of the form $G=G_{0} \rtimes \Gamma$, where $\Gamma \subseteq G$ normalizes some maximal torus $T$ and leaves invariant some Weyl chamber in $T$.

Proof. Fix a maximal torus $T \subseteq G_{0}$, and a Weyl chamber $C$. Set $N=N(T, C)$. As in Theorem 3.10, let $\mathcal{E}^{\prime}(N)$ be the set of subgroups $H \subseteq N$ of finite index such that $H / T$ is elementary but not of prime power order.

By Lemma 3.7(b), $\delta(H /[T, H])^{2}|| H / T||\left|\pi_{0}(G)\right|$ for each $H \subseteq N$ of finite index. So (1) follows from Theorem 3.10 .

(a) $\mathrm{rs}_{G}^{\mathrm{U}}$ is onto by Lemma 3.4 if $G$ is finite, and by (1) if $G$ is connected.

(b) If all elements of $\pi_{0}(G)=\pi_{0}(N)$ have prime power order, then $\mathcal{E}^{\prime}(N)=\emptyset$, and so $\mathrm{rs}_{G}^{\mathrm{U}}$ is onto by Theorem 3.10 .

(c) Note that $H_{2}(\Gamma)=0$ for any finite periodic group $\Gamma$. Hence, if $\pi_{0}(G)$ is periodic, then for any $H \in \mathcal{E}^{\prime}(N), H /[H, T] \cong T /[H, T] \times H / T$. So $\operatorname{rs}_{N}^{\mathrm{U}}$ and $\mathrm{rs}_{G}^{\mathrm{U}}$ are onto by Theorem 3.10 .

(e) The conditions on $\Gamma$ imply that $N$ is a semidirect product of $T$ with $\Gamma$, and hence that $\mathrm{rs}_{G}^{U}$ is onto by Theorem 3.10 .

(d) By $\left[\mathrm{Bo}, \S 4.10\right.$, Corollaire], the surjection $\operatorname{Aut}\left(G_{0}\right) \rightarrow \operatorname{Out}\left(G_{0}\right)$ is split by outer automorphisms which fix $T$ and $C$. Let $\Gamma \subseteq G$ be the subgroup of elements whose conjugation action lies in the image of any given splitting map. Then $G=G_{0} \rtimes \Gamma$ (since $G_{0} \cap \Gamma=Z\left(G_{0}\right)=1$ ); and so $\mathrm{rs}_{G}^{\mathrm{U}}$ is onto by (e).

We remark here that $G$ being a semidirect product $G_{0} \rtimes \Gamma$ does not in itself imply that $\mathrm{rs}_{G}^{\mathrm{U}}$ is onto. As an example, set

$$
G=C_{3} \times\left(\mathrm{SU}(2) \times_{C_{2}} Q(8)\right),
$$

where $C_{3}$ is cyclic of order $3, Q(8)$ is a quaternion group of order 8 , and the product is taken by identifying the central subgroups of order 2 in $\mathrm{SU}(2)$ and 
$Q(8)$. Then Theorem 3.10 applies to show that Coker $\left(\mathrm{rs}_{G}^{\mathrm{U}}\right)$ has exponent 2. But $\mathrm{SU}(2) \times{ }_{C_{2}} Q(8)$ is also a semidirect product of $\mathrm{SU}(2)$ with $C_{2} \times C_{2}$ : the splitting comes from the diagonal subgroup

$$
\langle(i, i)\rangle \times\langle(j, j)\rangle \subseteq Q(8) \times{ }_{C_{2}} Q(8) \subseteq \mathrm{SU}(2) \times{ }_{C_{2}} Q(8) .
$$

So far, we have dealt mostly with the case of unitary representations. The general conditions for $\mathrm{rs}_{G}^{\mathrm{O}}$ to be surjective seem to be much more complicated. For example, with a little more work, one can show that if $G$ is a central extension of a torus by a finite group, then $\mathrm{rs}_{G}^{\mathrm{O}}$ is onto if and only if $\mathrm{rs}_{G}^{\mathrm{U}}$ is onto. In contrast, the following example provides a simple way of constructing groups $G$ for which $\mathrm{rs}_{G}^{\mathrm{O}}$ is not onto but $\mathrm{rs}_{G}^{\mathrm{U}}$ is onto.

Example 3.12. Fix any pair $\left(G^{\prime}, V^{\prime}\right)$, where $G^{\prime}$ is a compact connected Lie group, and $V^{\prime}$ an irreducible $G^{\prime}$-representation of real type having the additional property that some central element $z \in Z\left(G^{\prime}\right)$ of order 2 acts on $V^{\prime}$ by $(-\mathrm{Id})$. Choose any odd prime power $n>1$, and set $G=G^{\prime} \times{ }_{C_{2}} Q(4 n)$ : the central product of $G^{\prime}$ with the quaternion group of order $4 n$, where $z$ is identified with the central element of $Q(4 n)$. Then $r s_{G}^{\mathrm{O}}$ is not onto.

Proof. Let $W$ be any effective irreducible representation of $Q(4 n)$, and set $V=$ $V^{\prime} \otimes_{\mathbb{C}} W$. Then $V$ is an irreducible $G$-representation of quaternion type, but its restriction to any $p$-toral subgroup of $G$ (for any prime $p$ ) has real type. In particular, $[V]$ represents an element of $\mathrm{RO}_{\mathcal{P}}(G)$; but since $\mathrm{rs}_{G}^{\mathrm{O}}$ and $\mathrm{rs}_{G}^{\mathrm{U}}$ are injective (all elements of $\pi_{0}(G) \cong D(2 n)$ have prime power order), it does not lie in the image of $\mathrm{rs}_{G}^{\mathrm{O}}$.

For example, we can take $G^{\prime}=\mathrm{SO}(2 m)$ for any $m \geq 2$, and let $V^{\prime}$ be the standard $G^{\prime}$-representation on $\mathbb{C}^{2 m}$. Set $G=G^{\prime} \times{ }_{C_{2}} Q(4 \bar{n})$, for some odd prime power $n \geq 3$. Then $\mathrm{rs}_{G}^{\mathrm{O}}: \mathrm{RO}(G) \rightarrow \mathrm{RO}_{\mathcal{P}}(G)$ fails to be onto, while $\mathrm{rs}_{G}^{\mathrm{U}}$ is onto (in fact, an isomorphism) by Corollary 3.11(b) (all elements of $\pi_{0}(G)$ have prime power order).

\section{References}

[Ad] J. F. Adams, Lectures on Lie groups, Benjamin, 1969.

[Bo] N. Bourbaki, Groupes et algèbres de Lie, Chapitre 9, Hermann, 1982.

[BtD] T. Bröcker and T. tom Dieck, Representations of compact Lie groups, Springer-Verlag, 1985.

[tD] T. tom Dieck, Transformation groups, de Gruyter, 1987.

[Dr] A. Dress, Induction and structure theorems for orthogonal representations of finite groups, Annals of Math. 102 (1975), 291-325.

[Fe] M. Feshbach, The transfer and compact Lie groups, Trans. A. M. S. 251 (1979), 139-169.

[JO] S. Jackowski and B. Oliver, Vector bundles over classifying spaces of compact Lie groups, Acta math. 176 (1996), 109-143. 
[Ol] R. Oliver, Whitehead groups of finite groups, London Math. Soc. Lecture Notes Series 132, Cambridge Univ. Press 1988.

[Seg] G. Segal, The representation ring of a compact Lie group, Publ. math. I. H. E. S. 34 (1968), 113-128.

[Ser] J.-P. Serre, Linear representations of finite groups, Springer-Verlag, 1977.

[Sn] V. Snaith, Explicit Brauer induction, Inventiones Math. 94 (1988), 455-478.

[Ta] M. Takeuchi, A remark on the character ring of a compact Lie group, J. Math. Soc. Japan 23 (1971), 662-675.

[Vo] D. Vogan, Unitary representations of reductive Lie groups, Annals of math. studies 118, Princeton Univ. Press, 1987.

Bob Oliver

URM 7539 du CNRS

Département de Mathématiques

Université Paris XIII

Av. J.-B. Clément

F-93430 Villetaneuse

e-mail: bob@math.univ-paris13.fr

(Received: April 22, 1996) 